\title{
Tunable Gap Plasmons in Gold Nanospheres Adsorbed into a pH- Responsive Polymer Film
}

Chih-Yu Jao,

Panupon Samaimongkola and

Hans D. Robinson*a

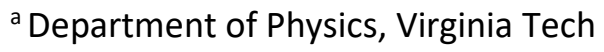

*Corresponding author.

Email: hansr@vt.edu

Tel.: +1-540-231-8732

Address:

Virginia Tech

Department of Physics

850 West Campus Drive

Blacksburg, VA 24061

This is the accepted manuscript of a paper published in the Journal of Colloid and Interface Science, accessible online at

http://dx.doi.org/10.1016/i.jcis.2019.06.018 


\begin{abstract}
Hypothesis

Plasmon nanorulers are exquisitely sensitive distance sensors that are based on the electromagnetic interaction between metal nanoparticles and surfaces. We hypothesize that nanorulers can act as quantitative probes of processes such as particle aggregation and adsorption, and deploy them to investigate particle adsorption onto stimulus-responsive polymer films. While such systems have previously been qualitatively investigated with plasmon nanorulers, our quantitative analysis should provide deeper insights.
\end{abstract}

\title{
Experiment
}

Gold nanospheres are adsorbed from solution onto $\mathrm{pH}$-responsive, amine-rich polyelectrolyte multilayer (PEM) films that are either directly deposited on a gold substrate or onto an intermediate selfassembled monolayer (SAM) of charged thiols. Fitting the optical scattering spectrum to a full-wave calculation, we quantify the sphere-substrate gap distance with good accuracy.

\section{Findings}

We find that the gold spheres partially embed into the PEMs rather than ride on top of them, and that although the amount of actuation of the spheres afforded by tuning the $\mathrm{pH}$ is well controlled, it is significantly smaller than the corresponding thickness changes in unstrained films. Further, the presence of a SAM below the PEM increases the amount of polymer in the PEM, except for the thickest and most highly charged films, where the SAM instead appears to displace from the area below the nanospheres.

Keywords: plasmonic nanoruler; responsive polymers, interface adsorption; particle embedding; polymer film

\section{Introduction}

The plasmon resonances in metal nanostructures are very sensitive to the geometry and composition of the metal and surrounding dielectric. This fact has been exploited in numerous sensing applications, starting with the commercially well-established SPR sensor [2-5], which is based on propagating surface plasmons in a thin metal film sensing changes in the dielectric environment as analytes bind to the film. The same principle has also been extensively studied in localized surface plasmon resonances (LSPRs) [6-8], where it has now reach such sensitivities that individual protein binding events can be detected [9].

Plasmon resonances are even more sensitive to the geometry of the metal, with the wavelength of an LSPR often sensitively dependent on sharp features and gap as small as a few nanometers. This poses a significant challenge to the fabrication of high-performance plasmonic devices, but can also be exploited in what are known as plasmon-based nanorulers [11-16], which can measure nm-length distances between metal features with sub-Ångström resolution.

The simplest structure that exhibits this sensitivity is probably a metal nanosphere separated from a metal plane by a thin dielectric spacer. Because of image charge effects, its optical properties are very similar to that of a metal particle dimer, but it is easier to fabricate and handle, and has therefore been studied extensively [1725]. The intensity enhancement of the plasmon mode inside the gap between particle and substrate is quite large in this configuration[24], which is why this system has also been studied as a substrate for surface enhanced Raman spectroscopy (SERS) [25-31].

In this article, we use the nanoruler principle to quantitatively investigate the adsorption of gold nanospheres onto, or as we will see, into, amine-rich polyelectrolyte multilayer (PEM) films, which have the property that they change thickness by up to several hundred percent in response to changes in ambient $\mathrm{pH}$. These films are a form of stimulus-responsive polymer structure [32], which is a class of materials that can take several forms, including brushes [33], microgels [34], and, like here, self-assembled multilayers [35]. They show repeatable and reversible conformational change in response to variables such as $\mathrm{pH}[1,36]$, temperature [37] or electric field [38], and are heavily studied for sensing and actuation applications at the nanoscale [32]. 
Our main results are that the nanospheres rather than ride on top of the film instead become partially embedded in it, and that modulating the film thickness through $\mathrm{pH}$ also actuates the positions of the nanospheres, albeit by a smaller amount. This type of tunability makes it possible to tune the properties of plasmonic devices and structures after fabrication, so that the specifications can be set more tightly than current fabrication technologies allow, and also enables new classes of dynamically adjustable devices and sensors.

PEMs are formed when oppositely charged polymers are alternately adsorbed onto a charged substrate $[35,39]$. In each deposition step, a dissolved polyelectrolyte adsorbs onto an oppositely charged substrate until the surface charge has been reversed, at which point the deposition stops. The substrate is then rinsed and immersed in a solution of the polyelectrolyte with opposite charge which again is deposited onto the surface until another charge reversal occurs. This process can be repeated as many times as desired to obtain a film of arbitrary thickness. Because each deposition step is self-limiting and relies primarily on Coulomb attraction, the thickness of the film is very well-controlled, allowing highly uniform films to be deposited rapidly across a large area, with conformal deposition over complicated geometries, and on a wide range of substrate materials.

Our PEMs are $\mathrm{pH}$-responsive and were deposited under moderately high $\mathrm{pH}$ conditions using the aminecontaining polymer poly(allylamine hydrochloride) (PAH), as the polycation. The polyanion was poly(styrene sulfonate) (PSS), and was deposited at the same $\mathrm{pH}$ as the $\mathrm{PAH}$. Since PAH is a weak base, it is only partially ionized at high $\mathrm{pH}$, and a larger quantity of polymer must therefore be deposited on the surface before charge reversal is accomplished and deposition stops. PSS on the other hand, is a strong acid and is fully ionized at all pH values, so a smaller amount of polymer is deposited in each step. As a result, a PAH/PSS PEM film deposited at $\mathrm{pH}$ values around 9 (i.e. near the solution $\mathrm{p} K_{a}$ of $\mathrm{PAH}$ ) are enriched in amines that are not coordinated with a sulfonate group. This means that when the ambient $\mathrm{pH}$ is modulated, the films respond by expelling or absorbing counterions accompanied by water as the amine groups loose or gain charge. As a result, the films swell or shrink by up to several hundred percent as $\mathrm{pH}$ is cycled between alkaline and acidic conditions $[1,36]$. The film thicknesses, particularly in the swelled state, can be in the range of $10 \mathrm{~s}$ of $\mathrm{nm}$, which at first blush would seem to make them unsuitable for a structure where the relevant distances are only a few $\mathrm{nm}$. But as we will show below, the unstrained film thickness does not correspond to the size of the surface-to-sphere gap for these films. The film is mechanically highly compliant, so the electrostatic attraction between the negatively charged sphere and positively charged film partially embeds the sphere in the film, particularly in its swelled state. When the film is compressed by the particles, water is expelled, reducing the screening around the positive charges in the films. The repulsive forces between those charges, as well as the reduction in conformational and other types of entropy, creates a counterforce from the film that eventually balances the attraction of the sphere to the film, but not until the film under the particles has been compressed to a fraction of its unstrained thickness.

This balance naturally varies with the swell state of the film, so the distance of the spheres to the substrate surface can be varied with $\mathrm{pH}$, although by less than the thickness variations in a free film under the same circumstances. As a result, we were able to tune the plasmon resonance wavelength by over $100 \mathrm{~nm}$, corresponding to vertical actuations of the spheres by as much as $10 \mathrm{~nm}$.

A good-sized body of literature already exists on combining plasmonic nanorulers with stimulus-responsive polymers to form both colloidal and surface-bound sensors [40-51]. The review by Tokarev and Minko [52] provides a good overview of this area. Much of this prior work is mainly qualitative in nature, and our present work is distinguished not just by the particular stimulusresponsive PEM used (which to our knowledge has not previously been studied with plasmon nanorulers) but the quantification of the size of the gap between sphere and surface, which helps shed light on the interaction of the nanosphere with the PEM and on the nature and structure of the film itself.

In some samples, we deposited a mercaptoundacanoic acid self-assembled monolayer (SAM) on the gold surface before the PEM deposition. We used this as a means of calibrating the plasmon nanoruler independently of our calculations, which works well for thin films prepared at low $\mathrm{pH}$. However, in some other cases, the addition of the SAM to the system may add a thickness to the film that is different from the known free thickness of the SAM. In particular, when the PEM is deposited on the SAM at the highest $\mathrm{pH}$ values, the apparent SAM thickness is at or near zero. We believe this is due either to the PEM films stripping away the SAM film during deposition, or to the adhesion forces between film and sphere being large enough to laterally displace the SAM layer from below the sphere. Such displacements have previously been observed in AFM experiments [53], although requiring forces that are about an order of magnitude larger than typical adhesion forces measured in PEMs. It is possible that the long interaction time (several hours in our case) means that significantly smaller forces are required to displace the SAM than in the sub-second times scale AFM measurements. 

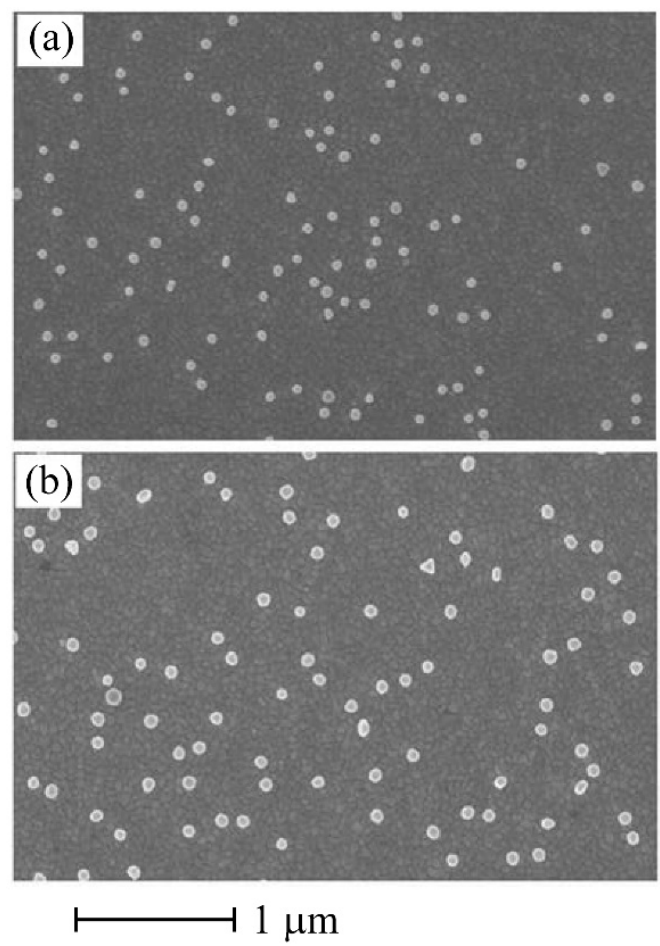

Figure 1. SEM micrographs of gold nanospheres, (a) $60 \mathrm{~nm}$ and (b) $80 \mathrm{~nm}$ in diameter, adsorbed onto $\mathrm{pH}$-responsive $\mathrm{PAH} / \mathrm{PSS}$ polyelectrolyte multilayers.

\section{Materials and Experimental Methods}

\subsection{Materials}

Suspensions of surfactant-free, citrate- and tannateterminated gold nanospheres with nominal diameters of $60 \mathrm{~nm}\left(2.6 \times 10^{10}\right.$ particles $\left./ \mathrm{ml}\right)$ and $80 \mathrm{~nm}\left(1.1 \times 10^{10}\right.$ particles $/ \mathrm{ml}$ ) were obtained from British Biocell International. Gold pellets, $99.999 \%$ pure and Titanium pellets, $99.995 \%$ pure were purchased from International Advanced Materials and Kurt J. Lesker Company, respectively. Polyallylamine hydrochloride (PAH) with $M_{W}$ $\sim 15,000$ and polystyrene sulfonate (PSS) with $M_{W} \sim 70,000$ were purchased from Sigma-Aldrich. Concentrated nitric and hydrochloric acid was purchased from Fisher Scientific.

\subsection{Sample Preparation}

All glassware used in sample preparation was cleaned by immersion in freshly prepared aqua regia $(3: 1 \mathrm{v} / \mathrm{v}$ conc. hydrochloric acid : conc. nitric acid) for 20 minutes and then rinsed with nanopure water and dried with a stream of dry nitrogen gas. Samples were prepared from 3 " $\times 1$ " microscope slides, cleaned in aqua regia and nanopure water, and mounted in an electron-beam evaporator where $100 \AA$ of $\mathrm{Ti}$ followed by $1400 \AA$ of $\mathrm{Au}$ was evaporated onto the slides. The polyelectrolyte multilayers were prepared as soon as possible after the slides were removed from the evaporator. In those cases

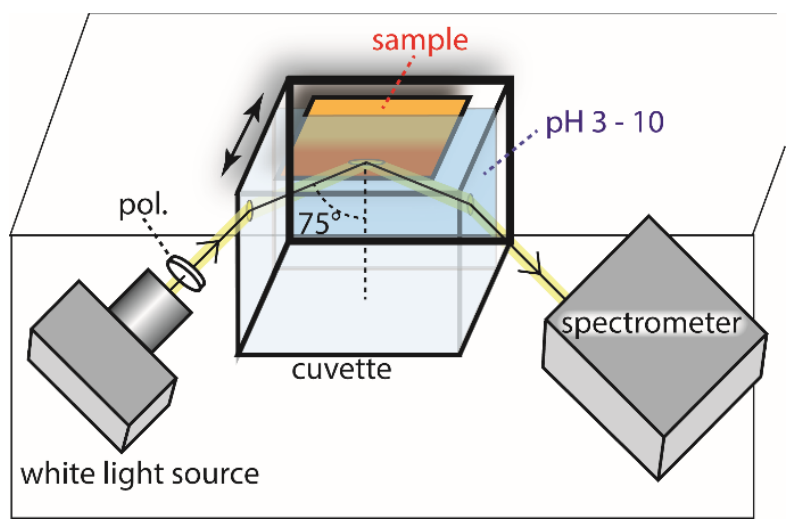

Figure 2. Top-view schematic of the optical setup. The sample (gold/red shading) is mounted on the inside back wall of a cuvette filled with $\mathrm{pH}$ adjusted water. Collimated light from a white light source impinged on the sample at $75^{\circ}$ incidence and was analyzed with an optical spectrometer. The cuvette was mounted on a vertical motion stage so that the reflection could be measured both in areas with and without nanospheres, and the extinction spectrum due to the spheres could be determined.

where a charged thiol-bound SAM was desired on the gold surface, the samples were first incubated for $16 \mathrm{~h}$ in a $1 \mathrm{mM}$ ethanolic solutions of the appropriate thiol, either 3-mercaptopropionic acid (MPA), 11-mercaptoundecanoic acid (MUA) or 16-mercaptohexadecanoic acid (MHDA). These solutions had first been acidified with a few drops of concentrated hydrochloric acid to ensure the formation of a dense thiol monolayer on the gold surface. The samples were then rinsed in nanopure water and dried with nitrogen gas.

For the fabrication of the PEMs, polyelectrolyte solutions with a $10 \mathrm{mM}$ repeat unit concentration were prepared from PAH $(0.94 \mathrm{~g} / \mathrm{l})$ and PSS $(1.83 \mathrm{~g} / \mathrm{l})$ by dissolving the dry polymer powder in nanopure water under gentle stirring for a minimum of $12 \mathrm{~h}$. We adjusted the $\mathrm{pH}$ of these solutions and of a nanopure water rinse bath to a chosen value between 8.5 and 9.5 by titration with $\mathrm{NaOH}$. The sample slides were then immersed in the PAH bath for 5 minutes followed by immersion under gentle agitation in the rinse bath for 2 minutes. The samples were subsequently immersed in the PSS bath for 5 minutes, which was also followed by immersion in the rinse bath. This resulted in what we term a one-bilayer film. The process can be repeated as desired to make thicker films. All samples were capped by a final PAH layer to ensure that the films carried a net positive charge under all relevant conditions, which means that all films consisted of a half-integer number of bilayers ranging from 0.5 to 4.5. To identify the different films, we adopt the nomenclature that [(PAH/PSS) 2.5 9.0] indicates a 2.5 bilayer film fabricated at $\mathrm{pH} 9.0$, terminated by a layer of PAH. After film fabrication, the slides were partially immersed without stirring in an as-received nanosphere suspension for 7 hours to induce adsorption of gold 


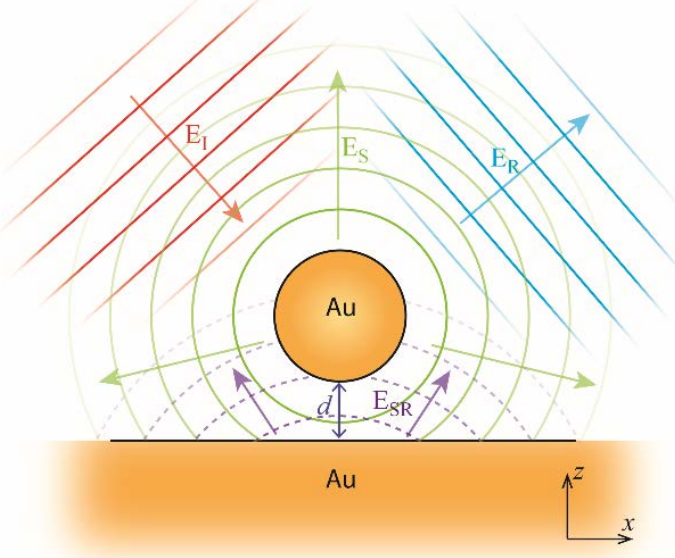

Figure 3. Schematic of field components used to calculate the scattering off a gold particle above a gold surface. $\mathbf{E}_{I}$-incident plane wave (red lines), $\mathbf{E}_{R}$-reflected plane wave (blue lines), $\mathbf{E}_{S}$-wave scattered by particle (green circles), $\mathbf{E}_{S R}-\mathbf{E}_{S}$ reflected from surface (dashed purple circle segments).

spheres on the immersed surface. Typical SEM micrographs of resulting samples are shown in Figure 1. Due to electrostatic repulsion between the spheres, aggregation between particles on the surface is minimal.

\subsection{Optical extinction measurements}

We collected optical extinction spectra from the samples with the setup shown schematically in Figure 2. Here, samples were mounted vertically and inward-facing on the back wall of a large cuvette with square cross section. p-polarized, collimated white light from a halogen lamp entered the cuvette at a $70^{\circ}$ angle to the cuvette side walls, and, since the cuvette contained $\mathrm{pH}$-adjusted water with $n=1.33$, reflected off the sample at a $75^{\circ}$ angle of incidence. The specularly reflected light was collected and fed into an optical spectrometer (Ocean Optics H2000CGUV-NIR) for analysis. The cuvette was mounted on a vertical motion stage so that different points on the sample could be measured without realigning the optics. Since each sample was fully coated with a PEM, but only partially coated with nanospheres, this made it simple to collect reflection spectra both from areas with $\left(I_{\mathrm{ns}}(\lambda)\right)$ and without $\left(I_{\text {film }}(\lambda)\right)$ adsorbed spheres. From this we calculate the extinction spectrum as $E(\lambda)=$ $\log _{10}\left(I_{\text {film }} / I_{\text {ns }}\right)$.

\section{Electromagnetic Modeling}

The scattering of light from the surface-adsorbed nanospheres was modeled with a full wave multi-pole expansion technique originally due to Bobbert and Vlieger [54], as extended by Johnson [55] and by Fucile el al. [56, 57]. This method is a version of T-matrix theory, which is widely used to model electromagnetic scattering by

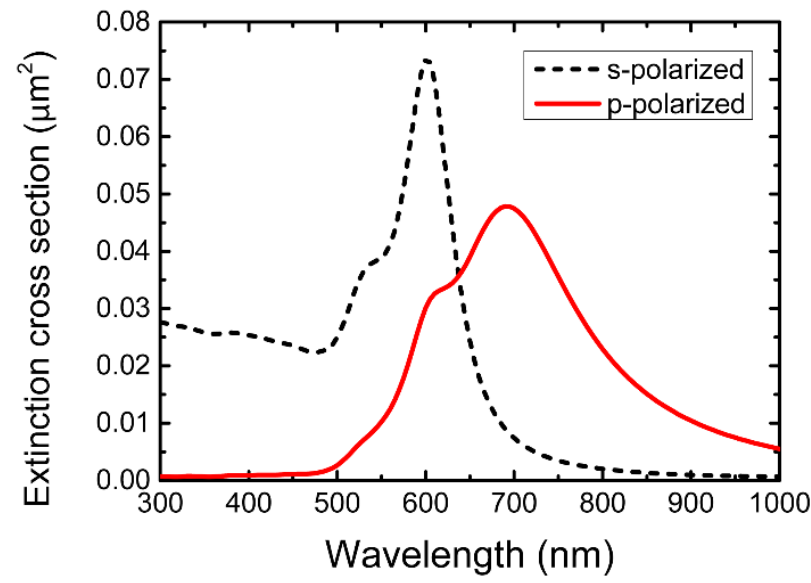

Figure 4. Calculated extinction spectra from $80 \mathrm{~nm}$ diameter gold spheres located $5 \mathrm{~nm}$ above a gold surface, in water. The angle of incidence is $75^{\circ}$. Spectra are shown for s-polarized (black dashed line) and p-polarized (red solid line) light.

nanoparticles $[58,59]$, including assemblies of plasmonic particles [60, 61]. A more detailed account of the technique and how it was applied in our case is given in the Supporting Information; here we confine ourselves to a brief overview.

In this method, the electromagnetic (EM) field is divided into four components as indicated in Figure 3: The plane wave that is incident on the sample $\left(\mathbf{E}_{I}\right)$, the reflection of this plane wave from the gold plane $\left(\mathbf{E}_{R}\right)$, the field that is scattered from the nanosphere $\left(\mathbf{E}_{S}\right)$, and its reflection from the gold surface $\left(\mathbf{E}_{S R}\right)$. We take the surface to be located in the $x y$-plane, with the sphere (with radius $a$ ) centered on the positive $z$-axis. The wave vector of the illuminating light is located in the $x z$-plane, and we denote the gold surfacesphere gap distance as $d$. It is the $\mathbf{E}_{S R}$ component that accounts for the interaction between the sphere and the substrate since it both results from $\mathbf{E}_{S}$ and is part of the incident field $\left(\mathbf{E}_{I}+\mathbf{E}_{R}+\mathbf{E}_{S R}\right)$ on the sphere which gives rise to $\mathbf{E}_{S}$. All the fields are decomposed into multipole expansions written as superpositions of Spherical Vector Wave Functions (SVWFs) centered on the sphere, which form a complete set of solutions to Maxwell's equations in a dielectric medium. Solving the problem then amounts to finding the coefficients of the multipole expansions that make the fields satisfy the boundary conditions on the surfaces of the sphere and the gold substrate. On the sphere, this is given by the conventional Mie scattering result [62], while on the substrate the SVWFs must be rewritten in terms of plane waves to properly account for the reflection. When all components of the near-field have been calculated, the far-field limit of $\mathbf{E}_{S}+\mathbf{E}_{S R}$ can be used to calculate the extinction crossection for the scattered light. This was done over a range of wavelengths to produce resonance spectra like those shown in Figure 4 and to 
which the experimental data can be fitted. The spectra in the figure are calculated for light incident on a gold sphere with a diameter $2 a=80 \mathrm{~nm}$ located $d=5 \mathrm{~nm}$ above the gold substrate and surrounded by water. The angle of incidence was $75^{\circ}$, same as in the experiments.

We see that resonances occur at different wavelengths depending on the polarization of the incident light. For spolarized light (incident electric field parallel to the substrate surface) we observe a resonance near $600 \mathrm{~nm}$, which is a distinct shift from the resonance at about 550 $\mathrm{nm}$ seen in the extinction spectrum from freely suspended gold nanospheres of the same size. For $p$ polarized light (electric field parallel to the plane of incidence) the resonance is even more strongly redshifted, with a maximum near $690 \mathrm{~nm}$.

The reason for this behavior can be gleaned from Figure 5, which plots the magnitude of the calculated EM field intensity surrounding the gold spheres in the $x z$-plane (the plane of incidence) both for an $80 \mathrm{~nm}$ gold sphere in an aqueous suspension (Figure $5(\mathrm{a})$ ) and one located $5 \mathrm{~nm}$ from a gold surface (Figure 5(b)). Both of the depicted modes are excited with a wavelength near the maximum optical response and, as indicated in the figure, with the same angle of incidence and polarization (which if Figure $5(b)$ is $p$-polarized relative to the substrate). For the freely suspended sphere, the electromagnetic field is essentially dipolar around the particle (although retardation effects shift the mode somewhat in the forward direction), and we will refer to this mode as a Mie plasmon. For the other sphere, the mode (known as a gap plasmon) is largely localized in the small gap volume between the sphere and the surface, indicating strong plasmonic coupling. As is typical of plasmonic systems, this coupling leads to a red shift of the resonance along with a significant enhancement of the field strength inside the gap. The latter point is illustrated by Figure 5(c), which shows that the intensity enhancement reaches values in excess of 1400 times in the gap plasmon, whereas it is only about 60 times for the Mie plasmon. The gap plasmon is strongly affected by the size $d$ of the gap between the sphere and the substrate, which makes it possible to determine $d$ with high precision by measuring the redshift between the Mie and gap plasmons, which is the essence of the operation of a plasmonic nanoruler.

In the above calculations, we used the dielectric function of gold as given by Johnson and Christy [63], and assumed that the dielectric medium surrounding the metal has a uniform index of refraction $n=1.33$, which is that of water. In our experiment, this neglects the optical effect of the polymer, which has a higher index of refraction, particularly in the compacted gap between sphere and substrate. As explained in the Supplementary Information, our method allows taking into account the polymer either as a film of thickness $d$ inserted between (a)
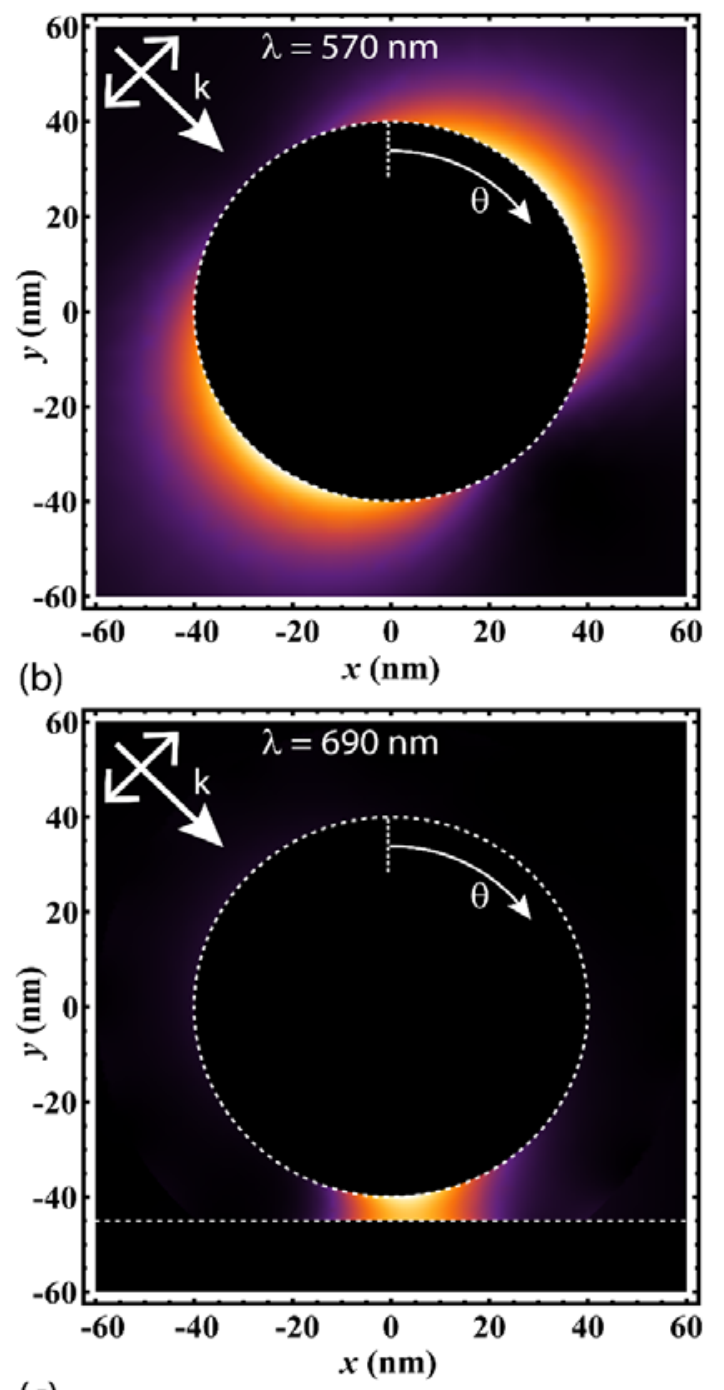

(c)

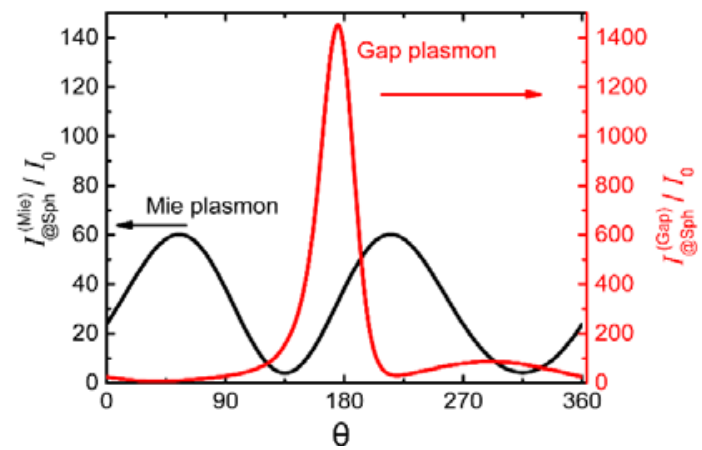

Figure 5. Calculated light intensities near $80 \mathrm{~nm}$ diameter gold spheres (outlined by dashed white lines) that are (a) suspended freely in water and illuminated at a wavelength of $570 \mathrm{~nm}$, (b) in water, positioned $5 \mathrm{~nm}$ from a gold surface (white dashed line) and illuminated at $690 \mathrm{~nm}$ wavelength. In both (a) and (b), the light is incident from the top left at a $45^{\circ}$ angle with the vertical and is polarized in the plane of the page, which also contains the center of the spheres. (c) plots the light intensity (relative to the incident intensity) along the surface of the spheres shown in (a) (in black-Mie plasmon) and (b) (in red-gap plasmon). The angle $\theta$ is taken clockwise from the upward vertical, as shown in the figure. 


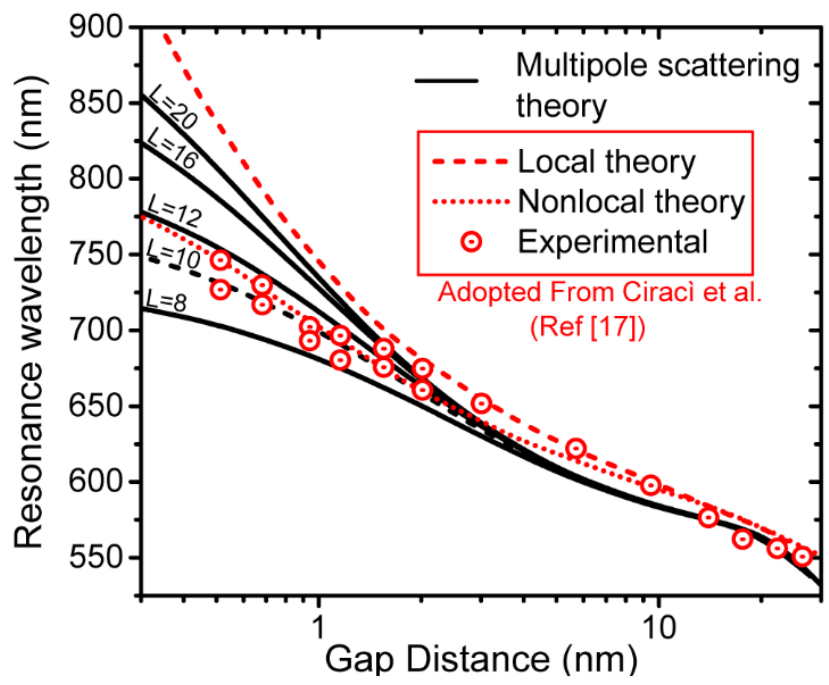

Figure 6. Plot of resonance wavelength vs gap distance for $60 \mathrm{~nm}$ diameter spheres in air separated from a planar gold substrate by an organic film. Data (circles) and theoretical results adopted from Ciraci et al. are shown in red, while our multipole calculation using the same starting parameters are shown as black lines for varying multipole truncation values $L$.

the substrate and the sphere, or by assuming that the entire sphere is fully embedded within the polymer film. In addition, numerical inaccuracies result from the need to truncate the multipole expansion at a finite $2 L$-pole, and from approximations in calculating the far-field behavior of $\mathbf{E}_{S R}$. Neither should have any appreciable impact on the calculated value of the plasmon resonance wavelength for the structures under consideration.

\section{Results and Discussion}

\subsection{Validation of the numerical model and initial results}

To validate our numerical model, we compare it to the calculations and experimental results obtained by Ciraci et al. [17], who studied a very similar system, consisting of $60 \mathrm{~nm}$ diameter gold spheres separated from a gold film by an organic spacer. Figure 6 shows their results in red compared to our model, shown as black lines, using different truncations $L$ in the multipole order. The main result of the Ciraci paper is their observation that at small gap distances $d$, the plasmon resonance is blueshifted with respect to its expected value obtained with local response theory. This is attributed to electron-electron interactions becoming significant in the plasmon hot spot when $d \lesssim 1 \mathrm{~nm}$, leading to a nonlocal dielectric function in the metal $[17,64]$. Interestingly, our multipole model fits reasonably well with the nonlocal behavior for distance larger than about $3 \AA$, if the calculation is cut off at a relatively small value of $L$, which for the $60 \mathrm{~nm}$ spheres here is approximately $L \approx 11$. This can be explained heuristically in the context of a hydrodynamic model of the electron gas nonlocality, where electronelectron interactions translate into an effective pressure [64] that tends to smooth out inhomogeneities in the electron density, reducing the maximum electron density in the hot spot and spreading electrons out over a larger volume around the gap. This in turn leads to a reduction of the proportion of high spatial frequencies in the charge distribution on the sphere and thereby in the electromagnetic field. Truncating the calculations at a finite $L$ amounts to imposing a cutoff in the angular frequency of the electromagnetic field, translating on a spherical particle to a corresponding cutoff in spatial frequency along the surface of the sphere. The cutoff therefore leads to a similar blue shift in the LSPR as when nonlocality is taken into account. This is quite convenient from the point of view of calculational efficiency, as the calculation time scales as $L^{3}$. We can then use a relatively small value of $L$, leading to faster computations without loss of accuracy.

When performing calculations for our own data, we need to scale the cutoff $L$ with the diameter of the particle to maintain the same cutoff in spatial frequency. Therefore, we will use a truncation of $L=14$ for the $80 \mathrm{~nm}$ diameter gold spheres we use in our experiments.

When we perform the fit, an additional source of uncertainty comes from a lack of knowledge of the exact conformation of the $\mathrm{pH}$-responsive films around the gold nanospheres. Unlike the fixed thickness PEM films used by Ciracì et al. [17] and in our own previous work,[65] these films are softer and more compliant, and we cannot a priori assume that the spheres are riding completely on top of the film. Equally likely, the spheres are partially or fully immersed within the film as this would substantially lower the electrostatic energy of the system. Fortunately, this uncertainty is relatively manageable since the index contrast between the film $(n \approx 1.45)$ and the water $(n=$ 1.33) in which it is immersed is relatively small. This is illustrated in Figure 7, where we plot the calculated plasmon resonance wavelength vs gap distance $\left(\lambda_{s p}(d)\right)$ for the limiting cases of the sphere riding on top of the film and the sphere being fully immersed within the film. We see that the two resulting traces are nearly parallel and fairly closely spaced, and we expect experimental data to fall somewhere between them.

Since the theoretically predicted $\lambda_{s p}^{\text {in film }}(d)$ and $\lambda_{s p}^{\text {on film }}(d)$ fall so close together, our data is not of sufficient accuracy to be of use in determining where between the two the best model lies in each case. For this reason, we will for the remainder of this paper adopt a model that is a weighted average of the two predictions:

$$
\lambda_{s p}(d)=\alpha \lambda_{s p}^{\text {in film }}(d)+(1-\alpha) \lambda_{s p}^{\text {on film }}(d),
$$




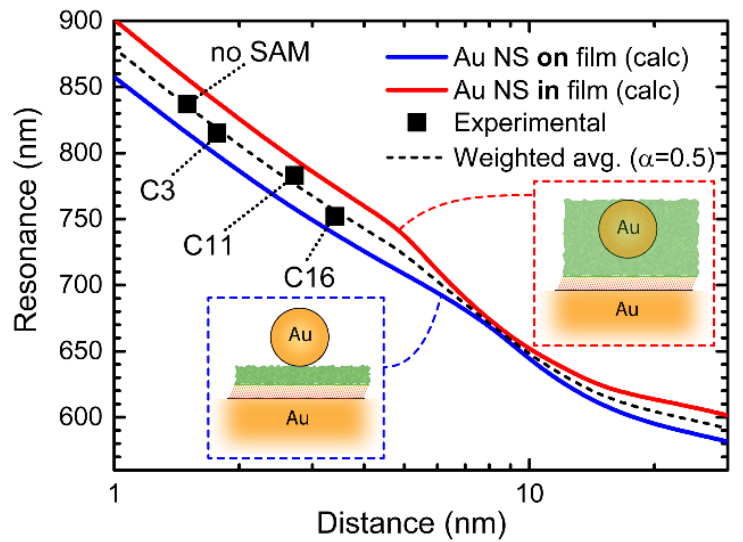

Figure 7. Theoretical plots and experimental data for plasmon resonances in $80 \mathrm{~nm}$ gold nanospheres adsorbed onto a film consisting of a single monolayer of PAH on top of SAMs of varying thicknesses. The solid lines indicate the calculated plasmon resonance wavelength for spheres separated from a gold surface by a dielectric with index of refraction $n_{2}=$ 1.45. The sample is immersed in water $(n=1.33)$. Calculations for $\mathrm{Au}$ nanospheres resting on the film $\left(n_{1}=1.33\right)$ and fully immersed in the film $\left(n_{2}=1.45\right)$ are shown in blue and red respectively. The black dashed line corresponds to an average of the two limiting cases with equal weight to each. Experimental data is indicated with black squares, for films assembled onto charged thiol SAMs of varying thickness ( 3 carbons, 11 carbons, and 16 carbons) or directly on the gold surface. The sole fitting parameter is the distance between the Au sphere and the SAM-polymer interface $\left(d_{\text {poly }}=\right.$ $1.48 \pm 0.29 \mathrm{~nm})$.

where for simplicity we chose $\alpha=0.50$, i.e. equal weight to the "on film" and "in film" cases. Given that $\alpha$ in practice will vary from sample to sample as well as with ambient $\mathrm{pH}$, this is a reasonable approach. $\lambda_{s p}(d)$ using these parameters is plotted in Figure 7 as a dashed black line, and the measured resonances are indicated by black squares.

As an initial sanity check on our model, we fabricated four different samples each consisting of a single PAH monolayer deposited at $\mathrm{pH} 9.0$, either directly on a gold film or onto negatively charged alkane thiol selfassembled monolayers (SAMs) of varying thicknesses that we first deposited onto the gold. Specifically, we used films assembled from MPA (indicated by C3 in Figure 7), MUA (C11), or MHDA (C16), which are known to form SAMs on planar gold with thicknesses of $0.27,1.22$, and $1.9 \mathrm{~nm}$, respectively [66]. The gap distance between gold sphere and gold surface is then given by the sum of the thickness of the SAM and a spacing due to the PEM film: $d=d_{S A M}+d_{\text {poly }}$. If we assume that $d_{\text {poly }}$ has the same value for all four samples, we can use it as a fitting parameter chosen to obtain a good fit between data and $\lambda_{s p}(d)$. As can be seen in Figure 7, the agreement is good for four data points, even though $d_{\text {poly }}$ is the sole fitting parameter, providing us with a partial validation of the model-not as strong as the agreement between our model and the results in Cirací et al. [17], but one that serves to validate its applicability to our experimental conditions. As discussed in detail in the Supplementary Information, fitting the data to our model without making any assumptions about the index of refraction of the film and medium gives us that $d_{\text {poly }}=1.48 \mathrm{~nm}$ with a $1 \sigma(68 \%)$ confidence interval of $\pm 0.21 \mathrm{~nm}( \pm 14 \%)$. The $2 \sigma(95 \%)$ confidence interval is $\pm 0.90 \mathrm{~nm}( \pm 60 \%)$, which means that we can with high confidence take $d_{\text {poly }}=1.5 \pm 0.90 \mathrm{~nm}$. If we also take into account published measurements of PEM indices of refraction, $[1,67-70]$ the confidence interval shrinks to ${ }_{-0.4}^{+0.7} \mathrm{~nm}\left(\begin{array}{l}+46 \\ -20\end{array}\right)$. This is the error due to uncertainties in the PEM index of refraction, and thus is a systematic error affecting all our results equally. The random error in our measurements will be much smaller-likely $5 \mathrm{~nm}$ or less in the resonance wavelength determination, corresponding to random measurement errors in $d$ of less than $\pm 10 \%$.

We need to mention that although we have high confidence in this error analysis, there are uncertainties we are not able to quantify. For example, the model assumes perfectly spherical and monodisperse particles, which we can see from Figure 1 is clearly an idealization. Surface roughness of the substrate and effects such as chemical interface damping (CID) [71, 72] are also not accounted for. Complete certainty regarding our results would therefore require measurements of $d$ with independent techniques, and in their absence we cannot exclude errors larger than those just quoted. Still, since our work is fully consistent with the large body of prior work on plasmon nanorulers, and because the totality of all the fitted data tells a consistent story about the system under study, we believe it is unlikely the unknown errors in our estimates are large enough to undermine the conclusions reached in the discussion section below. Beyond enabling an error analysis, the data in Figure 7 also validates our assumption that $d_{\text {poly }}$ of a single PAH layer is approximately the same whether the polymer is deposited on a charged SAM or directly on a gold surface, or it would not have been possible to simultaneously fit all four data points in Figure 7 to $\lambda_{s p}(d)$. As we will show below, this assumption does not hold for thicker PEMs, but it is sufficiently valid for the single PAH layer that all four data points can be included in the fit in Figure 7.

The data in Figure 7 was taken at pH 10 when the films are in their deswollen state. One objective of this paper is to demonstrate that the gap distance between the gold sphere and surface can be manipulated repeatably and with good precision across a large range, which is accomplished by changing the ambient $\mathrm{pH}$. Figure 8 shows measured p-polarized extinction spectra (as solid lines) in reflection from a $\left[(\mathrm{PAH} / \mathrm{PSS})_{3.5}\right.$ 9.3] film with $60 \mathrm{~nm}$ 


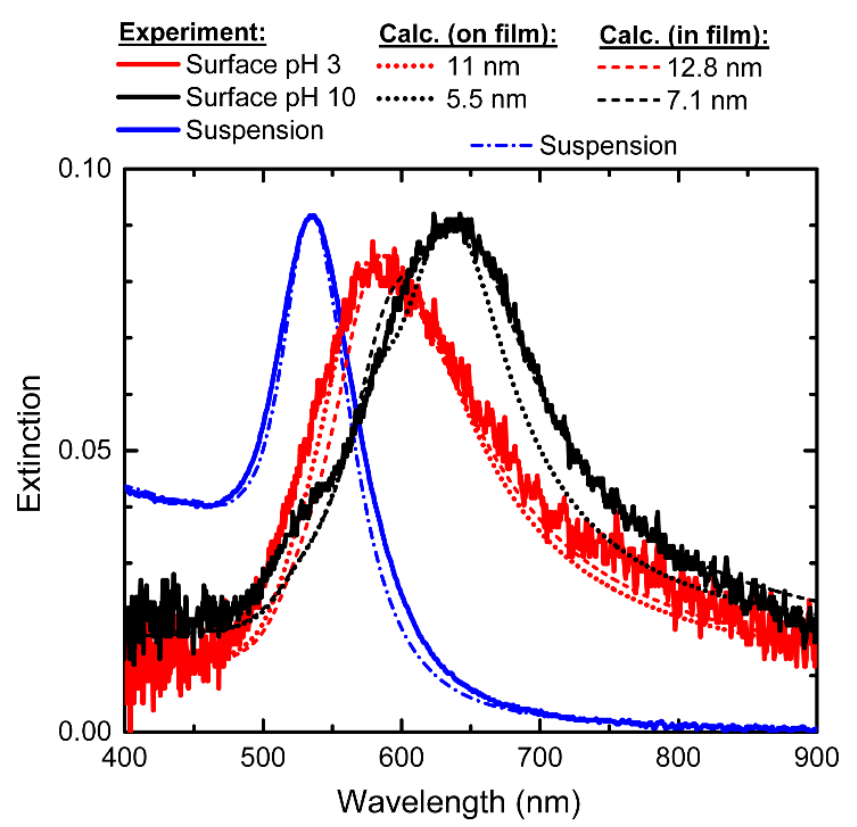

Figure 8. Extinction spectra from $60 \mathrm{~nm}$ diameter gold particles in an aqueous suspension (blue curves, peaked near $530 \mathrm{~nm}$ ) and adsorbed onto a 3.5 bilayer $\mathrm{PAH} / \mathrm{PSS}$ film prepared at $\mathrm{pH}$ 9.3, shown at an ambient $\mathrm{pH}$ of 3 , where the film is fully swelled (red curves, peaked near $580 \mathrm{~nm}$ ) and at $\mathrm{pH}$ 10 , where the film is in its deswelled state (black curves, peaked near 650 $\mathrm{nm}$ ). The solid curves are data while the broken curves are theoretical fits using Mie theory in the case of the suspended particles (dash-dotted line), and T-matrix theory for the adsorbed particles. In the latter case, the dotted curves correspond to the assumption that the particles are adsorbed on top of the film with no penetration, and the dashed lines to the assumption that particles are fully immersed inside the film.

diameter gold nanospheres adsorbed onto it, taken at $\mathrm{pH}$ 3 , when the film is in its fully swelled state, and at $\mathrm{pH} 10$, when it is fully deswelled. For comparison, an extinction spectrum of the same spheres suspended in water is also shown. At either $\mathrm{pH}$ value, the plasmon resonances seen in extinction spectra from the surface are substantially redshifted compared to the resonance seen in the suspension. As expected, the red shift is significantly larger at pH 10, when the film is at its thinnest.

Theoretical fits with our simulations are plotted in Figure 8 as broken lines. In the case of the suspension, Mie scattering theory was used to produce the fit, with the overall amplitude as the only free parameter. The gap plasmon curves shown as dotted lines are calculated under the assumption that the gold particles rest on a flat polymer film with no part of the particle below the top surface (particle on film), while the dashed curves assume that the particles are fully submersed within the film (particle in film). These two cases of course correspond to the blue and red curves in Figure 7. The two fitting parameters in these cases are the overall amplitude and the size $d$ of the gap. We see that the when the $\mathrm{pH}$ of the film is changed from 10 to 3 , corresponding respectively to the deswelled and swelled states of the film, the gap plasmon undergoes a large blue shift as the particles are moved farther from the substrate surface. The model indicates that during this process, the gap distance is increased from $6.3_{-1.4}^{+3.0} \mathrm{~nm}$ to $11.8 \pm{ }_{2.6}^{5.5} \mathrm{~nm}$, where most of the uncertainty stems from the lack of knowledge of the degree of particle immersion in the film $(\alpha)$. The fits also show relatively good agreement between the calculated and measured peak shapes, supporting the validity of the theoretical model.

The measured peaks are somewhat wider than the calculated ones, but this is to be expected as this is an ensemble measurement, where inevitable variations in particle size and gap distance are bound to cause some inhomogeneous broadening of the observed plasmon resonances. The modest broadening in Figure 8 , much of which may in fact be due to CID [71, 72], is typical for the vast majority of the samples, indicating a good uniformity of the films, leading to consistent embedding of gold spheres, so that the plasmon resonance peak position provides a good estimate of the gap distance for most spheres in a sample. The exceptions are the thicker (3.5 and 4.5 bilayers) films prepared at $\mathrm{pH}$ 9.5, where the broadening is more substantial. This is not unexpected, since PAH/PSS films made at $\mathrm{pH} 9.5$ are at the edge of becoming unstable and known to exhibit substantial roughness [73], which then leads to a corresponding variation in gap distance of the adsorbed particles.

\subsection{Measuring particle embedding}

To characterize the interaction of the gold nanospheres with the $\mathrm{pH}$-responsive films, we fabricated a number of different samples with varying thicknesses and preparation $\mathrm{pH}$. We chose 8.5, 9.0, 9.3, and 9.5 for the preparation $\mathrm{pH}$ values, with two samples of each kind fabricated at $\mathrm{pH} 9.0$ to test reproducibility. At each preparation $\mathrm{pH}$, samples with $0.5,1.5,2.5,3.5$, and 4.5 bilayers of PAH/PSS were fabricated (the 0.5 bilayer film consists only of a single PAH layer). Each of the samples was fabricated in two versions, one where the PEM was deposited directly on the gold substrate, and one where a SAM of MUA was deposited first, followed by the PEM.

After gold spheres had been adsorbed onto all 50 samples, the extinction spectrum of each was measured both in the swollen ( $\mathrm{pH} \mathrm{3}$ ) and deswollen (or shrunken) $(\mathrm{pH} 10)$ states. The main peak in all spectra was fit to a Pearson type IV distribution with the LevenbergMarquardt algorithm, with the sole exception of the [(PAH/PSS) 4.5 9.5] sample without underlying SAM, where the peak was so broad that we judged that assigning an average gap distance would be of questionable value. The peak position was converted into a gap distance using Eq. 1 with $\alpha=0.5$. In what follows, we denote with $d_{P E M}$ the 
(a)
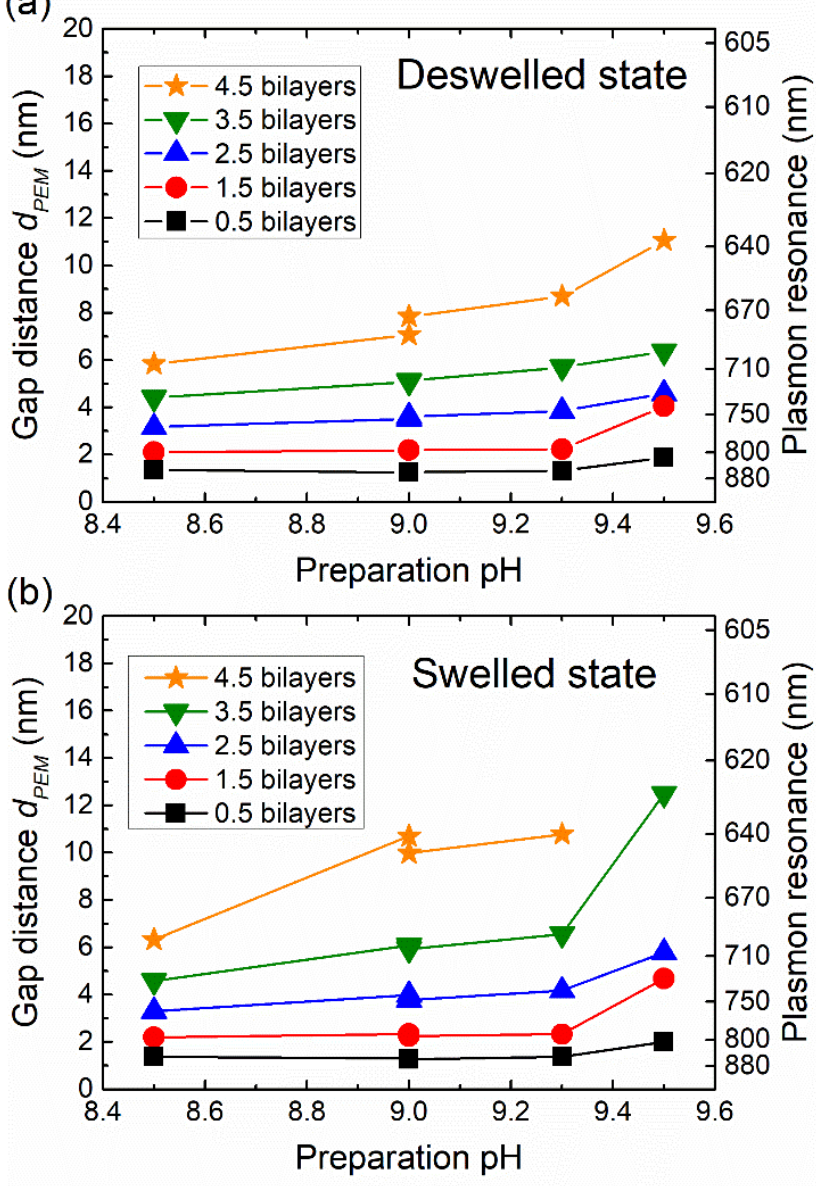

Figure 9. Plot of particle-substrate gap distances $d_{P E M}$, calculated from extinction spectra, in samples consisting of $80 \mathrm{~nm}$ diameter gold nanoparticles adsorbed onto PAH/PSS films fabricated directly on a gold surface, prepared at different $\mathrm{pH}$ values and containing different numbers of polyelectrolyte bilayers. Gap distances are shown both for films in their deswelled state (ambient pH 10-panel (a)) and films in their swelled state (pH 3-panel (b)). The gap distances are calculated from the plasmon resonance peak wavelengths, which are shown on the right side of the graphs.

gap distance in those films where the PEM was deposited directly on the substrate, and with $d_{P E M / S A M}$ the distance in those films where the PEM is deposited on an underlying SAM layer.

These results are plotted in Figure 9 for samples without a SAM layer and in Figure 10 for samples with a SAM layer below the PEM. We will first focus on the data in Figure 9, as it concerns films that lack a thiol SAM and is the simplest to interpret. We see, as expected, that the gap distance $d$ increases with the number of deposited polyelectrolyte bilayers. By taking the difference in $d$ between films with different numbers of bilayers, we can deduce the effective contribution to $d$ of each PSS/PAH bilayer as well as of the initial PAH monolayer. This contribution is the smallest for the first layers, and then rises monotonically through the fourth bilayer, consistent
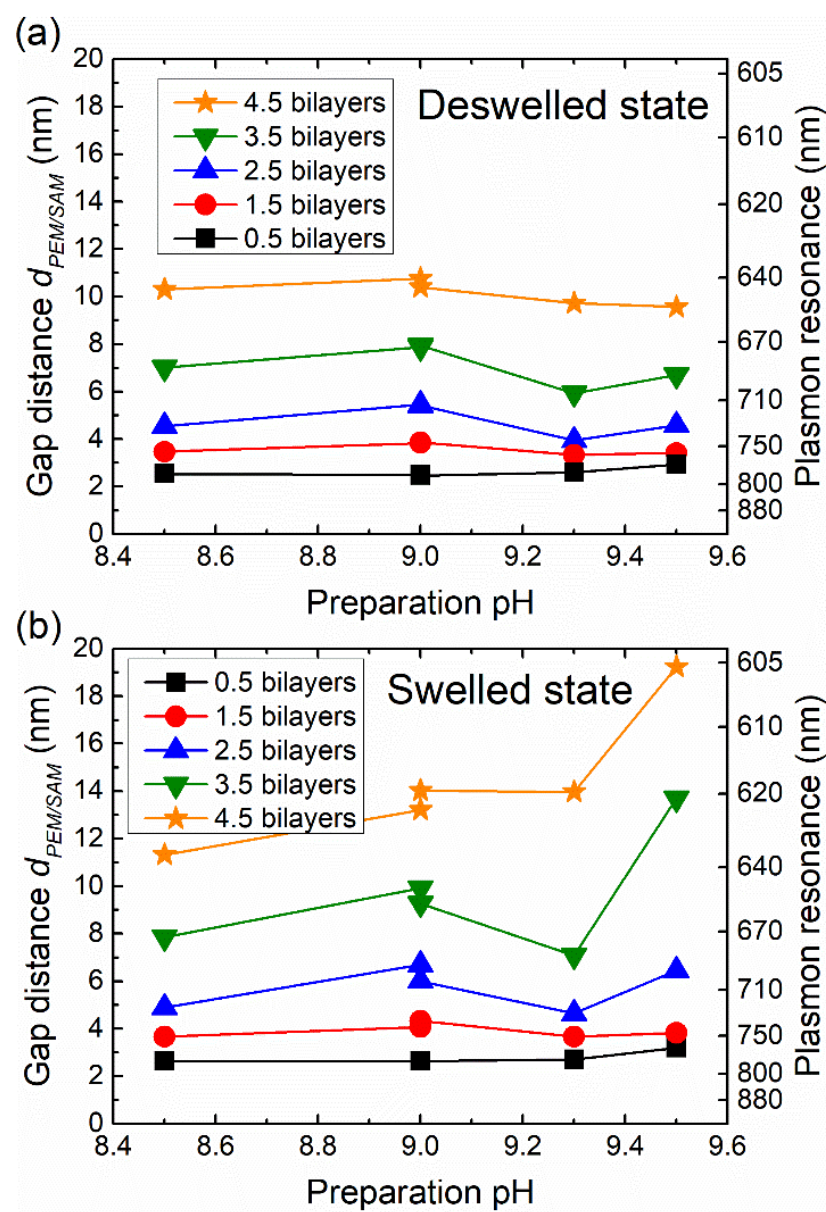

Figure 10. Plots of gap distances $d_{P E M / S A M}$ from optical extinction data as in Figure 9, but from samples where a mercaptoundecanoic acid SAM was assembled on the gold substrate prior to PEM film deposition.

with prior reports [74]. This is illustrated in Figure 11, where the contribution of the initial PAH layer and all four subsequent bilayers in the films prepared at $\mathrm{pH} 9.3$ are plotted. It is instructive to compare these values to the bilayer thickness in unstrained films of the same type, which are indicated in red in the figure, and taken from Itano et al. [1] as well as from our own previously published measurements (Ashry et al.) [10]. The value from Itano comes from ellipsometry measurements on $\sim 50 \mathrm{~nm}$ thick films in the dry state, while the Ashry number comes from AFM measurements on 0.5 and 1.5 bilayer films in the wet deswelled state. In spite of the differences in film condition and thickness, the measured bilayer thicknesses are very similar in the two published reports ( $4.6 \mathrm{~nm}$ and $4.5 \mathrm{~nm}$ respectively). This comparison is extended to all SAM-free samples in Table I, and shows that the gold nanoparticles are partially embedded within the films as the value of $d$ in each case scales roughly with the unstrained film thickness, so that film compressions below the gold sphere in the range of about $50 \%-60 \%$ is observed in all cases. These values are sufficiently large 
Table I. Comparison of effective contribution of each bilayer to the sphere-surface gap distance $d$ with known values for per-bilayer thickness in unstrained films, as well as the swell seen in unstrained films and the nominal amine excess that causes it (calculated assuming a PAH p $K_{a}$ of 8.8 ).

\begin{tabular}{cccccc}
\hline Preparation $\mathrm{pH}$ & $\begin{array}{c}\text { Nominal amine } \\
\text { excess in film }\end{array}$ & $\begin{array}{c}\text { Average per bilayer } \\
\text { contribution to } d(\mathrm{~nm})\end{array}$ & $\begin{array}{c}\text { Bilayer thickness in } \\
\text { unstrained films }(\mathrm{nm})\end{array}$ & $\begin{array}{c}\text { Est. film } \\
\text { compression }\end{array}$ & $\begin{array}{c}\text { Swell in unstrained } \\
\text { films }\end{array}$ \\
\hline 7.5 & $5 \%$ & - & $0.8^{\mathrm{a}}$ & - & - \\
8.5 & $50 \%$ & 1.1 & $2.5^{\mathrm{a}}$ & $55 \%$ & $230 \%^{\mathrm{a}}$ \\
9.0 & $160 \%$ & 1.6 & $4.0^{\mathrm{a}}$ & $60 \%$ & $440 \%$ \\
9.3 & $320 \%$ & 1.8 & $4.6^{\mathrm{a}}, 4.5^{\mathrm{b}}$ & $61 \%$ & $470 \%^{\mathrm{a}}$ \\
9.5 & $500 \%$ & 2.3 & $4.9^{\mathrm{a}}$ & $53 \%$ & $500 \%$ \\
\hline
\end{tabular}

a Ref. [1] (Itano et al.)

b Ref. [10] (Ashry et al.)

that they are unlikely to be explained by uncertainties or inaccuracies in our model or by measurement errors (indicated by the error bars in Figure 11) and we conclude that the gold nanospheres are in fact embedded into the PEM films in all our samples.

The penetration of a nanoparticle into a polymer film comes at the cost of conformational entropy of the polymer, leading to an entropic expelling force. In our films, this force counteracts the Coulomb force that pulls the negatively charged particle into the positively charged film, and limits the depth of penetration. The gap distance is in other words set by a balance between the Coulomb force and the entropic forces (which at small gap distances will also include hydration and osmotic forces) as well as any mechanical forces that may occur due to the compression of the polymer film. This mechanism qualitatively explains our observation of partial embedding. At the same time, we need to note that previous work measuring gap distances in similarly sized gold nanospheres adsorbed onto positively charged PEM films did not report any significant compression of the film [17]. Likely, this is because the earlier work used films assembled under conditions of near-neutral $\mathrm{pH}$ that lead to PEMs that have a lower charge density $[1,73]$, and because PAH/PSS films soften markedly when exposed to high $\mathrm{pH}$ values like those used in this work [75]. These differences will lead respectively to larger expelling forces and smaller Coulomb attraction in films prepared under neutral conditions, so that the degree of film compression in those cases may have been sufficiently small so as to be easily overlooked. As discussed in section S2 of the Supplementary Information, it is also possible that Ciraci et al. did not notice particle embedding even though it was present, because they overestimated the index of refraction of their films.

\subsection{Nanoparticle actuation}

Comparing Figure 9(a) and 9(b) (as well as Figure 10(a) and $10(b))$, it is clear that in all cases, swelling of the films leads to actuation of the gold nanoparticles away from the substrate. The size of this motion was derived from
Figure 9 and plotted in Figure 12, and varies substantially depending on the film preparation. Due to the high sensitivity of the plasmonic nanoruler, we are able to detect motions on the order of $0.2 \AA$ in the thinnest, least responsive films, up to motions as large as $7 \mathrm{~nm}$ in the thickest films prepared at $\mathrm{pH}$ 9.5. In a relative sense, this corresponds to changes in the gap distance $d$ that vary between $1.2 \%$ and $96 \%$. The actuations give rise to substantial and easily detected shifts in the gap plasmon resonances, but the physical particle displacements are significantly smaller than the corresponding actuation in unstrained PEM film thicknesses, where $\mathrm{pH}$-induced swelling leads to thickness changes in excess of $200 \%$ in films prepared at $\mathrm{pH} 9.0$ and above (See Table I) [1, 36]. Given the large Coulomb forces that embed the particles within the film combined with the high mechanical compliance of the uncrosslinked polymer, a discrepancy between film swelling and particle motion is not

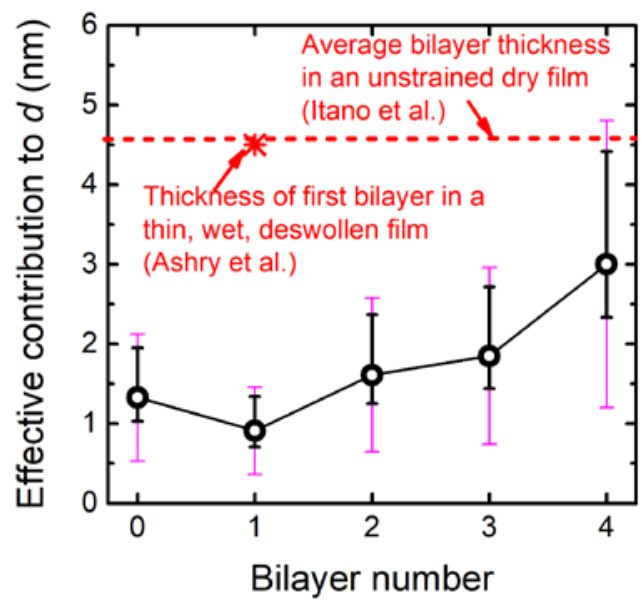

Figure 11. Plot of the effective contribution of each PSS/PAH bilayer (except that "bilayer" 0 is taken to consist only of the initially deposited $\mathrm{PAH}$ monolayer) to the gap $d$ for films prepared at $\mathrm{pH} 9.3$, extracted from the data in Figure 9(a). As comparison, previously published bilayer thicknesses in identically prepared films are shown in red, with data taken from Itano et al. [1] and Ashry et al. [10] The black error bars indicate errors taking into account the known index of refraction range in our PEM films, while the magenta error bars reflect errors calculated using the experimental data in Fig 6. 

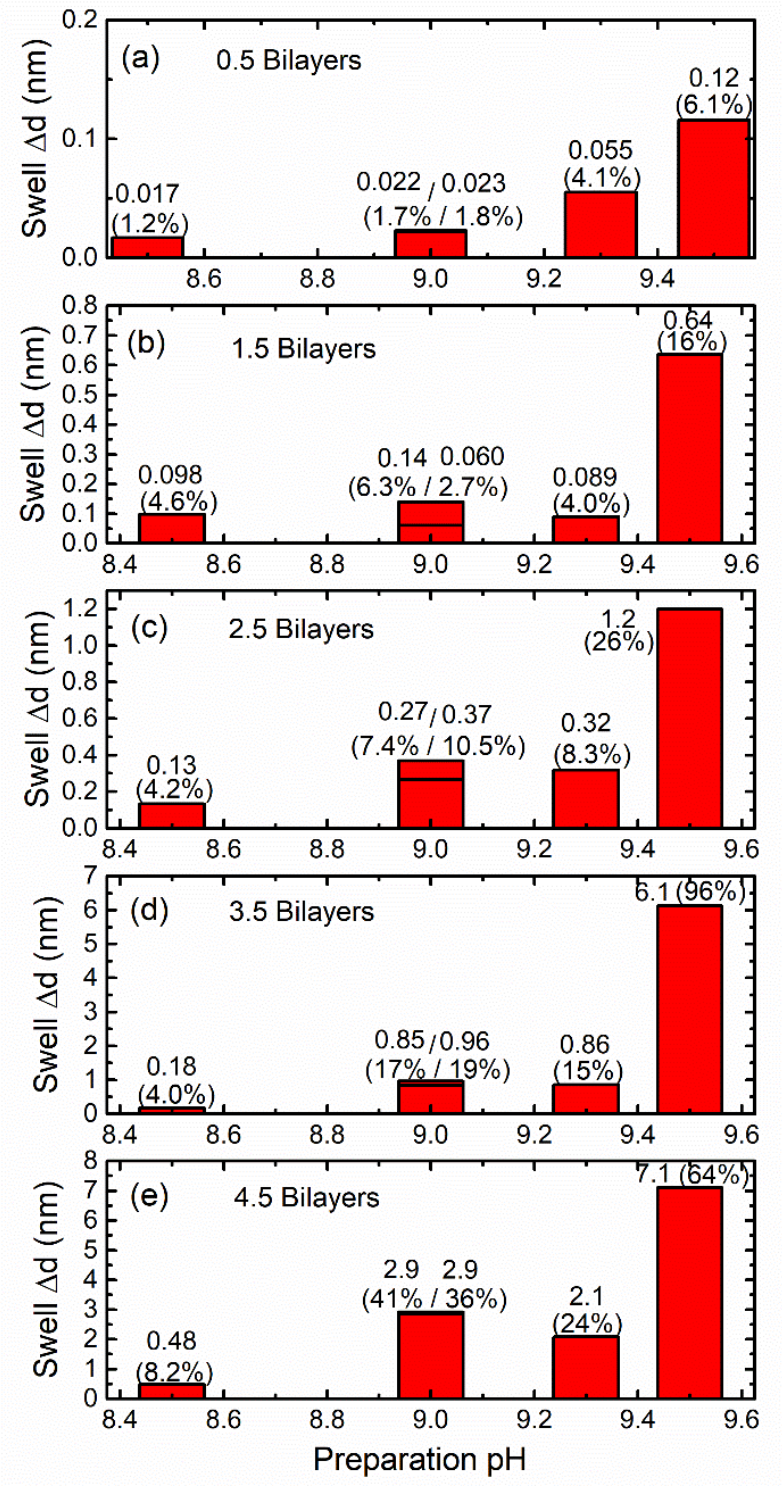

Figure 12. The bar graphs show the amount of nanoparticle actuation in $\mathrm{nm}$ away from the surface in various PEM films (without underlying SAM) as the ambient $\mathrm{pH}$ is changed from 3 to 10 , causing the films to swell. The corresponding percentage changes in $d$ are displayed in parentheses. Two numbers are shown at a preparation $\mathrm{pH}$ of 9.0 since two different sample sets were prepared at that $\mathrm{pH}$.

surprising. At the same time, the attractive forces are not so large that they completely suppress particle actuation, and we see the particles move whenever the underlying film swells, although we cannot necessarily use the thickness and degree of swelling of unstrained films as a guide to the size and tunability of $d$.

When we examine the trends in Figure 12, two things stand out. First, although the swell $\Delta d$ generally increases with preparation $\mathrm{pH}$, as expected, the films prepared at $\mathrm{pH} 9.5$ really stand out in terms of their ability to actuate the particles, in a way not seen in the swelling of unstrained films, where, as shown in Table I, the

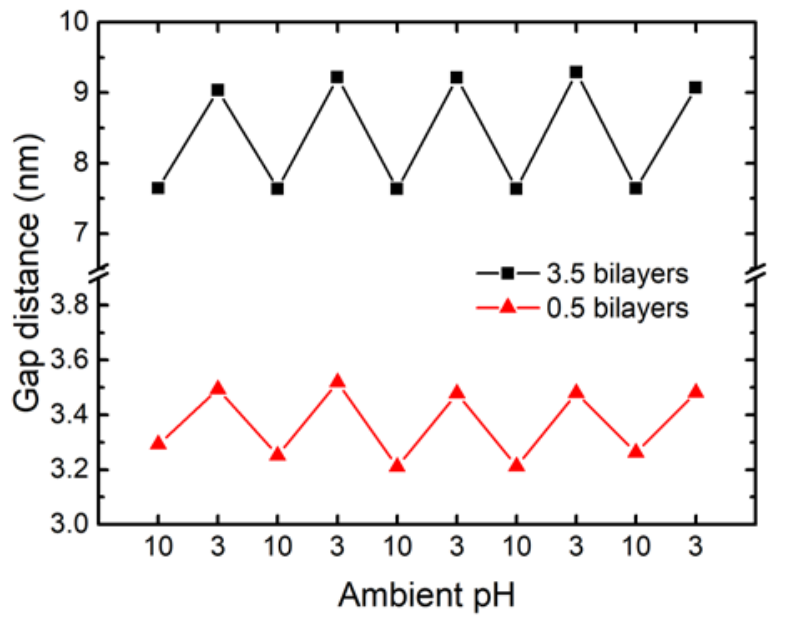

Figure 13. Measured particle-substrate gap distances in samples where the dielectric spacer consisted of a single PAH layer (red triangles) and a 3.5 bilayer PAH/PSS film (black squares) as the samples are repeatedly cycled between $\mathrm{pH} 3$ and 10. Both films were deposited at $\mathrm{pH} 9.5$.

differences in swell between films prepared at $\mathrm{pH}$ 9.0, 9.3, and 9.5 is relatively small. Some of this difference can likely be attributed to the much larger fraction of excess amines in the films fabricated at $\mathrm{pH} 9.5$, as also shown in Table I. Second, not only is $\Delta d$ larger for thicker films, as one might expect, but it grows significantly faster with bilayer number than the film thickness. This manifests in Figure 12 as a steady increase in the percentage numbers when scanning from panel (a) (0.5 bilayers) to panel (e) 4.5 bilayers. Both of these observations indicate that the competency of the film in actuating the adsorbed particles grows significantly as more PAH is deposited on the substrate.

With an eye to applications, it is also important to note that the $\mathrm{pH}$-induced actuation of the nanoparticles is reversible multiple times. This is shown in Figure 13, where two samples ( 0.5 and 3.5 bilayer films prepared at $\mathrm{pH}$ 9.5) are alternately exposed to $\mathrm{pH} 3$ and $\mathrm{pH} 10$ conditions, leading to repeatable actuation of the gold particles of approximately $0.3 \mathrm{~nm}$ and $1.5 \mathrm{~nm}$ respectively, with no hint of drift or other change in the size of motion that exceed the noise of the measurement during the 9 actuation steps performed on each sample.

\subsection{Effects of Mercaptoundecanoic acid SAM}

Next, we turn our attention to the effect of inserting a SAM made from a negatively charged alkanethiol (MUA) between the gold surface and the PEM. To first order, we expect the effect of the SAM to be an increase in the distance that equals the thickness of the SAM $d_{S A M}$, which for MUA is known to be about $1.22 \mathrm{~nm}$ [66]. In other words, $d_{P E M}=d_{\text {poly }}$, and $d_{P E M / S A M}=d_{S A M}+d_{\text {poly }}$, so the difference of the two thicknesses should equal $d_{S A M}$ 
(a)

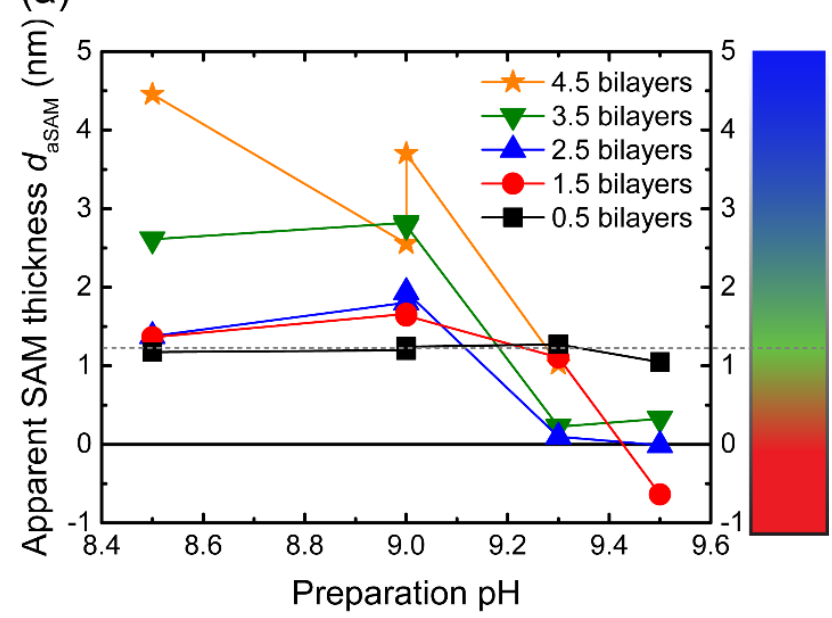

(b)

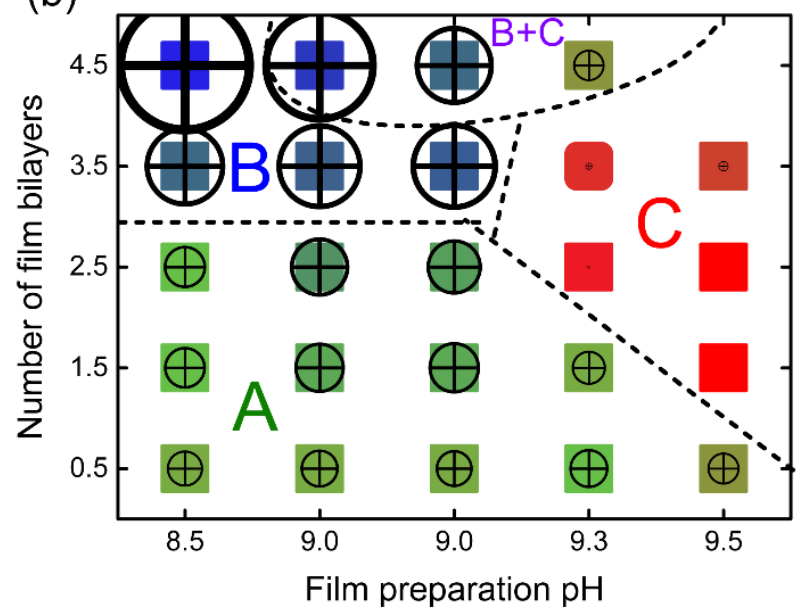

Figure 14. (a) Plot of the difference in gap distance $d_{a S A M}$ between films prepared with and without a mercaptoundecanoic acid SAM below the PEM, which can be interpreted as a measure of the effective thickness of the SAM. The dashed grey line indicates the $1.2 \mathrm{~nm}$ thickness of a freestanding SAM on gold. (b) Diagram of the same data as shown in (a) plotted vs bilayer number and preparation $\mathrm{pH}$ for each of the films, where the imputed SAM thickness is indicated both by the size of the circle symbol and the hue of the colored square at each point in the matrix. The hue is selected using the color gradient shown along the right axis of (a). The films can be divided into three categories as indicated in the figure: $\mathrm{A}-d_{\text {aSAM }}$ is about the same as or slightly larger than the thickness of a freestanding SAM (green tones). $\mathrm{B}-d_{\text {aSAM }}$ distinctly larger than the thickness of a freestanding SAM (blue tones). $\mathrm{C}-d_{\text {aSAM }}$ is approximately zero (red tones).

so long as $d_{\text {poly }}$ is the same for both films. Comparing Figure 9 and 10, we see that the reality is more complicated than that, with the gap distance $d$ in some cases appearing to decrease with preparation $\mathrm{pH}$ when the SAM is present. To see the effect of the SAM more clearly, we plot in Figure 14 the apparent SAM thickness $d_{\text {aSAM }}=d_{P E M / S A M}-d_{P E M}$, obtained as the differences between the data in Figures 9(a) and 10(a). Calculating the difference between swollen films in Figures $9(b)$ and 10 (b) yields similar results.

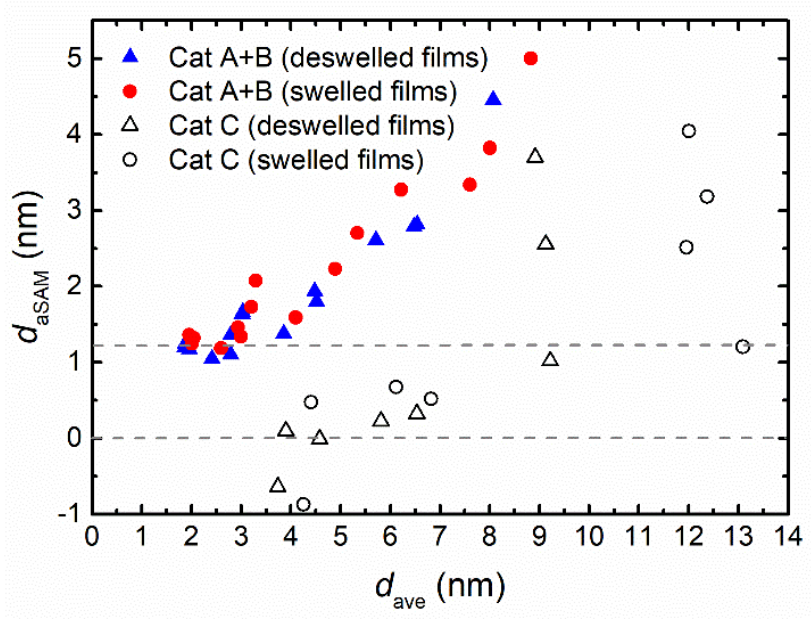

Figure 15. Apparent SAM thickness $d_{\text {aSAM }}$ plotted vs average $d_{\text {ave }}$ of the thicknesses of the films deposited with and without an underlying SAM layer. The grey dashed lines indicate $d_{a S A M}=0 \mathrm{~nm}$ and $1.2 \mathrm{~nm}$, the known thickness of a freestanding MUA SAM.

It is clear from Figure 14, that it is only in the relatively thinner films fabricated at lower $\mathrm{pH}$ values where our naïve expectation that $d_{a S A M} \approx d_{S A M}$ is borne out. Specifically, $d_{\text {aSAM }}$ approximately equals $1.2 \mathrm{~nm}$ (indicated by a dashed line in the figure) for all the 0.5 bilayer films. We also arrive at values of $d_{a S A M}$ somewhat larger than $1.2 \mathrm{~nm}$ for the 1.5 bilayer films fabricated at $\mathrm{pH} 9.3$ and lower, and the 2.5 bilayer films fabricated at $\mathrm{pH} 9.0$ and lower. This type of behavior is labeled as category ' $\mathrm{A}$ ' in Figure $14(\mathrm{~b})$. In addition, 3.5 and 4.5 bilayer films prepared at $\mathrm{pH} 9.0$ and 8.5 give rise to an apparent SAM thickness that is appreciably larger than 1.2 $\mathrm{nm}$ (category ' $\mathrm{B}$ '). In contrast, films with 1.5 bilayers or greater prepared at the highest $\mathrm{pH}$ values, appear to have $d_{\text {aSAM }}$ approximately equal to zero (category ' $\mathrm{C}$ '). Classification of the [(PAH/PSS $]_{4.5}$ 9.0] and [(PAH/PSS $]_{4.5}$ 9.3] films is uncertain, and these films may represent a boundary case between category $B$ and $C$.

Turning first to the films in category $A$ and $B$, a more careful inspection of the data reveals that these films in fact fall on a continuum, with the excess of $d_{a S A M}$ over the true SAM thickness growing progressively larger as the total film thickness grows. This is shown in Figure 15, where $d_{\text {aSAM }}$ values in category $\mathrm{A}$ and $\mathrm{B}$ are plotted vs the average $d_{\text {ave }}=\frac{1}{2}\left(d_{S A M / P E M}+d_{P E M}\right)$ of the data from which $d_{a S A M}$ is calculated. We can see that $d_{a S A M}$ grows nearly linearly with $d_{\text {ave }}$, starting near $1.2 \mathrm{~nm}$ for the thinnest films, growing to about $5 \mathrm{~nm}$ for the thickest ones.

As explained in detail in the Supplementary Information, this effect cannot be explained by inaccuracies in our model, because any model that leads to a constant value $d_{a S A M}$ leads to an unphysical relationship between $d$ and 
$\lambda$, incompatible not only with our own model, but to all previous observations of this kind of system (See Figure S2 and discussion in section S2.3 of the Supplementary Information).

To explain the discrepancy between $d_{a S A M}$ and the free SAM layer thickness, we note that the thickness of the first few bilayers in a PEM depends strongly on the nature of the underlying surface. In particular, Caruso et al. have reported that the initial bilayers of a PAH/PSS films are thinner and less consistent when grown directly on bare gold compared to when it is grown on a gold surface capped with a negatively charged SAM [74]. In other words, the amount of polymer deposited per bilayer in the SAM/PEM films is greater than in the plain PEM films, leading to $d_{a S A M}>d_{S A M}$, with the difference negligible for the very thinnest films, but growing steadily as additional polyelectrolyte is deposited on the surface. Specifically, our data indicates that the SAM layer increases the amount of polymer deposited (and thereby $d_{\text {poly }}$ ) in films with more than one bilayer by between $10 \%$ and $60 \%$, with the highest increases seen in the thickest ( 4.5 bilayer) films.

Films fabricated at the highest $\mathrm{pH}$ (category $\mathrm{C}$ films) exhibit the opposite effect in that rather than increasing the film thickness, the presence of an underlying SAM appears to have no material effect on the gap distance. This is perhaps the most surprising result of this study, so it bears pointing out that it does not depend on our model or any faults or inaccuracies it might contain. Instead, it rests solely on the observation that for the category $C$ samples, the plasmon resonance wavelength is nearly identical for samples prepared with and without an underlying SAM, which means we must conclude that $d_{a S A M} \approx 0$ regardless of model for the plasmon ruler.

It seems unlikely that adding a SAM to the surface would reduce the amount of deposited polymer in the category $C$ samples when it has the opposite effect in the category $B$ case, so the explanation for our result must reside with the SAM. It is possible that the PEMs assembled at high $\mathrm{pH}$ through some mechanism simply removes the SAM from the surface. Another possibility is that the SAM in these films is pushed aside due the force between sphere and substrate. The thickest films assembled at the highest $\mathrm{pH}$ values hold the greatest charge density, which leads to the highest coulomb forces, consistent with the observation that larger bilayer count and higher preparation $\mathrm{pH}$ are required for the apparent SAM thickness to be zero. Previous work by Carpick and Salmeron indicate that to promptly collapse a dense SAM with a small radius AFM tip, surface pressures on the order of $1 \mathrm{GPa}$ are required [53]. For our spheres, this would correspond to adhesion forces $F$ on the order of 30 $\mathrm{nN}$, or $F / R=750 \mathrm{mN} / \mathrm{m}$ if rescaled by the tip radius $R$ (which is relevant due to the Derjaguin approximation, where $F / R$ equals $2 \pi$ times the interaction energy per area). Similar forces are also required to shave thiol layers off with an AFM tip under lateral movement [76]. By comparison, the maximum pull-off forces measured with the colloidal probe technique for PEM films have typically been in the range of $4-20 \mathrm{mN} / \mathrm{m}$ [77-79], so it is unlikely that the attraction between PEM and sphere is sufficiently strong to provide the forces observed by Salmeron et al. However, the spheres are in contact with the SAM for a very long time-several hours-compared to the subsecond interaction time in the AFM experiments, and it is conceivable that over this time scale, substantially smaller forces are required to displace the SAM from below the sphere. We finally note that one report exists of surface adhesion energies as high as $300 \mathrm{~mJ} / \mathrm{m}^{2}$ in swellable PEMs [80], although much of this energy may be due to very short range interactions that have no impact on the pressure below the nanosphere embedded in the film.

\section{Conclusions}

In this paper, we have studied the use of plasmonic nanorulers to characterize the interaction between gold nanospheres and $\mathrm{pH}$-responsive PEMs. For distances less than about $20 \mathrm{~nm}$, the accuracy of this nanoruler measurement is at the sub-nm level or better. It is worth noting that the scattering from the particles is also sufficiently strong that the plasmon spectrum of individual particles can be measured directly $[11,20,81$, 82], at least for spheres larger than about $50 \mathrm{~nm}$ in diameter. This makes gap plasmons an excellent technique for probing the interaction between nanoparticles and organic films.

In contrast to prior work of this type, which has mostly been qualitative in nature [40-52], our modeling provides quantitative information on the separation between the nanospheres and the substrate. This has allowed us to draw several conclusions about the interaction between the particles and the PEM film. Notably, we have shown that (1) the nanospheres embed a significant amount into the PEM rather than ride on top of it, (2) nanoparticle actuation is repeatable and well-controlled, but notably smaller than the thickness changes seen in an unstrained film, (3) depositing PEM on a charged SAM leads to increased polymer deposition in the PEM, which we can quantify, except that (4) in the thickest and most highly charged PEMs, the SAM is instead desorbed or displaced from the region below the nanosphere.

Our modeling includes an error analysis that accounts for uncertainties in the distribution of index of refraction in the volume surrounding the nanospheres as they interact with the organic film. This enhances the utility of the technique, as it demonstrates that we can draw 
quantitative conclusions about a system even without detailed knowledge of the dielectric environment that is being probed.

In addition to using a gap plasmon nanoruler to investigate organic films, this work also suggests that it is possible to do the reverse and use tunable organic films to precisely regulate the spectral position of plasmon resonances. This is important because current fabrication technologies often do not allow plasmonic and other nano-optical structures to be fabricated with sufficient precision, and also because dynamically tunable devices are of greater utility than static ones. A good example of this is the currently heavily studied area of plasmonic metasurfaces [83], where reconfigurability is an important goal [84].

\section{Acknowledgements}

This work was performed with support from the Institute for Critical Technology and Applied Science (ICTAS) at Virginia Tech and was supported by a grant from the National Science Foundation under agreement DMR1006753. The authors also acknowledge BBI Solutions for graciously providing gold nanosphere samples used in this work.

\section{References}

1. K. Itano, J. Y. Choi, and M. F. Rubner, "Mechanism of the $\mathrm{pH}$-induced discontinuous swelling/deswelling transitions of poly(allylamine hydrochloride)-containing polyelectrolyte multilayer films," Macromol. 38, 34503460 (2005).

2. J. Homola, S. S. Yee, and G. Gauglitz, "Surface plasmon resonance sensors: review," Sens. Actuators, B 54, 3-15 (1999).

3. X. D. Hoa, A. G. Kirk, and M. Tabrizian, "Towards integrated and sensitive surface plasmon resonance biosensors: A review of recent progress," Biosens. Bioelectron. 23, 151-160 (2007).

4. A. Shalabney, and I. Abdulhalim, "Sensitivity-enhancement methods for surface plasmon sensors," Laser Photonics Rev. 5, 571-606 (2011).

5. S. Zeng, D. Baillargeat, H.-P. Ho, and K.-T. Yong, "Nanomaterials enhanced surface plasmon resonance for biological and chemical sensing applications," Chem. Soc. Rev. 43, 3426-3452 (2014).

6. J. Zhao, X. Zhang, C. R. Yonzon, A. J. Haes, and R. P. Van Duyne, "Localized surface plasmon resonance biosensors," Nanomed. 1, 219-228 (2006).
7. G. J. Nusz, A. C. Curry, S. M. Marinakos, A. Wax, and A. Chilkoti, "Rational Selection of Gold Nanorod Geometry for Label-Free Plasmonic Biosensors, " ACS Nano 3, 795-806 (2009).

8. S. Szunerits, and R. Boukherroub, "Sensing using localised surface plasmon resonance sensors," Chem. Commun. 48, 8999-9010 (2012).

9. P. Zijlstra, P. M. R. Paulo, and M. Orrit, "Optical detection of single non-absorbing molecules using the surface plasmon resonance of a gold nanorod," Nature Nanotechnol. 7, 379-382 (2012).

10. I. Ashry, B. G. Zhang, M. B. Khalifa, J. A. Calderone, W. L. Santos, J. R. Heflin, H. D. Robinson, and Y. Xu, "Fluorescence lifetime based characterization of active and tunable plasmonic nanostructures," Opt. Express 22, 20720-20726 (2014).

11. C. Sönnichsen, B. M. Reinhard, J. Liphardt, and A. P. Alivisatos, "A molecular ruler based on plasmon coupling of single gold and silver nanoparticles," Nat. Biotechnol. 23, 741-745 (2005).

12. K. H. Su, Q. H. Wei, X. Zhang, J. J. Mock, D. R. Smith, and S. Schultz, "Interparticle Coupling Effects on Plasmon Resonances of Nanogold Particles," Nano Lett. 3, 10871090 (2003).

13. B. M. Reinhard, S. Sheikholeslami, A. Mastroianni, A. P. Alivisatos, and J. Liphardt, "Use of plasmon coupling to reveal the dynamics of DNA bending and cleavage by single EcoRV restriction enzymes," Proc. Nat. Acad. Sci. 104, 2667-2672 (2007).

14. D. S. Sebba, J. J. Mock, D. R. Smith, T. H. LaBean, and A. A. Lazarides, "Reconfigurable Core-Satellite Nanoassemblies as Molecularly-Driven Plasmonic Switches," Nano Lett. 8, 1803-1808 (2008).

15. Y.-w. Jun, S. Sheikholeslami, D. R. Hostetter, C. Tajon, C. S. Craik, and A. P. Alivisatos, "Continuous imaging of plasmon rulers in live cells reveals early-stage caspase-3 activation at the single-molecule level," Proc. Nat. Acad. Sci. 106, 17735-17740 (2009).

16. G. L. Liu, Y. Yin, S. Kunchakarra, B. Mukherjee, D. Gerion, S. D. Jett, D. G. Bear, J. W. Gray, A. P. Alivisatos, L. P. Lee, and F. F. Chen, "A nanoplasmonic molecular ruler for measuring nuclease activity and DNA footprinting," Nature Nanotechnol. 1, 47-52 (2006).

17. C. Ciraci, R. T. Hill, J. J. Mock, Y. Urzhumov, A. I. FernándezDomínguez, S. A. Maier, J. B. Pendry, A. Chilkoti, and D. R. Smith, "Probing the Ultimate Limits of Plasmonic Enhancement," Science 337, 1072-1074 (2012).

18. R. T. Hill, J. J. Mock, A. Hucknall, S. D. Wolter, N. M. Jokerst, D. R. Smith, and A. Chilkoti, "Plasmon Ruler with Angstrom Length Resolution," ACS Nano 6, 9237-9246 (2012).

19. T. Okamoto, and I. Yamaguchi, "Optical Absorption Study of the Surface Plasmon Resonance in Gold Nanoparticles 
Immobilized onto a Gold Substrate by Self-Assembly Technique," J. Phys. Chem. B 107, 10321-10324 (2003).

20. M. Hu, A. Ghoshal, M. Marquez, and P. G. Kik, "Single Particle Spectroscopy Study of Metal-Film-Induced Tuning of Silver Nanoparticle Plasmon Resonancest," J. Phys. Chem. C 114, 7509-7514 (2010).

21. N. Yamamoto, S. Ohtani, and F. J. García de Abajo, "Gap and Mie Plasmons in Individual Silver Nanospheres near a Silver Surface," Nano Lett. 11, 91-95 (2010).

22. S. Mubeen, S. Zhang, N. Kim, S. Lee, S. Krämer, H. Xu, and M. Moskovits, "Plasmonic Properties of Gold Nanoparticles Separated from a Gold Mirror by an Ultrathin Oxide," Nano Lett. 12, 2088-2094 (2012).

23. L. He, E. A. Smith, M. J. Natan, and C. D. Keating, "The Distance-Dependence of Colloidal Au-Amplified Surface Plasmon Resonance," J. Phys. Chem. B 108, 10973-10980 (2004).

24. K. Wang, E. Schonbrun, and K. B. Crozier, "Propulsion of Gold Nanoparticles with Surface Plasmon Polaritons: Evidence of Enhanced Optical Force from Near-Field Coupling between Gold Particle and Gold Film," Nano Lett. 9, 2623-2629 (2009).

25. J. D. Driskell, R. J. Lipert, and M. D. Porter, "Labeled Gold Nanoparticles Immobilized at Smooth Metallic Substrates: Systematic Investigation of Surface Plasmon Resonance and Surface-Enhanced Raman Scattering," J. Phys. Chem. B 110, 17444-17451 (2006).

26. J. K. Daniels, and G. Chumanov, "Nanoparticle-Mirror Sandwich Substrates for Surface-Enhanced Raman Scattering," J. Phys. Chem. B 109, 17936-17942 (2005).

27. K. Kim, and J. K. Yoon, "Raman Scattering of 4Aminobenzenethiol Sandwiched between Ag/Au Nanoparticle and Macroscopically Smooth Au Substrate," J. Phys. Chem. B 109, 20731-20736 (2005).

28. S.-Y. Chen, J. J. Mock, R. T. Hill, A. Chilkoti, D. R. Smith, and A. A. Lazarides, "Gold Nanoparticles on Polarizable Surfaces as Raman Scattering Antennas," ACS Nano 4, 6535-6546 (2010).

29. N. H. Kim, S. J. Lee, and M. Moskovits, "Aptamer-Mediated Surface-Enhanced Raman Spectroscopy Intensity Amplification," Nano Lett. 10, 4181-4185 (2010).

30. L. Rodríguez-Lorenzo, R. A. Álvarez-Puebla, I. PastorizaSantos, S. Mazzucco, O. Stéphan, M. Kociak, L. M. LizMarzán, and F. J. García de Abajo, "Zeptomol Detection Through Controlled Ultrasensitive Surface-Enhanced Raman Scattering," J. Am. Chem. Soc. 131, 4616-4618 (2009).

31. B. Liu, A. Blaszczyk, M. Mayor, and T. Wandlowski, "RedoxSwitching in a Viologen-type Adlayer: An Electrochemical Shell-Isolated Nanoparticle Enhanced Raman Spectroscopy Study on Au(111)-(1×1) Single Crystal Electrodes," ACS Nano 5, 5662-5672 (2011).
32. M. A. C. Stuart, W. T. S. Huck, J. Genzer, M. Muller, C. Ober, M. Stamm, G. B. Sukhorukov, I. Szleifer, V. V. Tsukruk, M. Urban, F. Winnik, S. Zauscher, I. Luzinov, and S. Minko, "Emerging applications of stimuli-responsive polymer materials," Nature Mater. 9, 101-113 (2010).

33. R. Barbey, L. Lavanant, D. Paripovic, N. Schüwer, C. Sugnaux, S. Tugulu, and H.-A. Klok, "Polymer Brushes via Surface-Initiated Controlled Radical Polymerization: Synthesis, Characterization, Properties, and Applications," Chem. Rev. 109, 5437-5527 (2009).

34. T. Ngai, S. H. Behrens, and H. Auweter, "Novel emulsions stabilized by $\mathrm{pH}$ and temperature sensitive microgels," Chem. Commun., 331-333 (2005).

35. G. Decher, J. D. Hong, and J. Schmitt, "Buildup of ultrathin multilayer films by a self-assembly process: III. Consecutively alternating adsorption of anionic and cationic polyelectrolytes on charged surfaces," Thin Solid Films 210-211, 831-835 (1992).

36. J. Hiller, and M. F. Rubner, "Reversible molecular memory and $\mathrm{pH}$-switchable swelling transitions in polyelectrolyte multilayers," Macromol. 36, 4078-4083 (2003).

37. R. Liu, M. Fraylich, and B. Saunders, "Thermoresponsive copolymers: from fundamental studies to applications," Colloid. Polym. Sci. 287, 627-643 (2009).

38. F. Zhou, P. M. Biesheuvel, E.-Y. Choi, W. Shu, R. Poetes, U. Steiner, and W. T. S. Huck, "Polyelectrolyte Brush Amplified Electroactuation of Microcantilevers," Nano Lett. 8, 725-730 (2008).

39. P. Bertrand, A. Jonas, A. Laschewsky, and R. Legras, "Ultrathin polymer coatings by complexation of polyelectrolytes at interfaces: suitable materials, structure and properties," Macromol. Rapid Commun. 21, 319-348 (2000).

40. T. Yin, X. Liu, J. Wang, Y. An, Z. Zhang, and L. Shi, "Thermosensitive mixed shell polymeric micelles decorated with gold nanoparticles at the outmost surface: tunable surface plasmon resonance and enhanced catalytic properties with excellent colloidal stability," RSC Advances 5, 47458-47465 (2015).

41. S. Gupta, P. Uhlmann, M. Agrawal, S. Chapuis, U. Oertel, and M. Stamm, "Immobilization of Silver Nanoparticles on Responsive Polymer Brushes," Macromol. 41, 2874-2879 (2008).

42. I. Tokareva, S. Minko, J. H. Fendler, and E. Hutter, "Nanosensors based on responsive polymer brushes and gold nanoparticle enhanced transmission surface plasmon resonance spectroscopy," J. Am. Chem. Soc. 126, 1595015951 (2004).

43. I. Tokarev, I. Tokareva, and S. Minko, "Optical Nanosensor Platform Operating in Near-Physiological pH Range via Polymer-Brush-Mediated Plasmon Coupling," ACS Appl. Mat. Interfaces 3, 143-146 (2011). 
44. S. Z. Nergiz, and S. Singamaneni, "Reversible Tuning of Plasmon Coupling in Gold Nanoparticle Chains Using Ultrathin Responsive Polymer Film," ACS Appl. Mat. Interfaces 3, 945-951 (2011).

45. H. Gehan, L. Fillaud, M. M. Chehimi, J. Aubard, A. Hohenau, N. Felidj, and C. Mangeney, "Thermo-induced Electromagnetic Coupling in Gold/Polymer Hybrid Plasmonic Structures Probed by Surface-Enhanced Raman Scattering," ACS Nano 4, 6491-6500 (2010).

46. M. K. Gupta, S. Chang, S. Singamaneni, L. F. Drummy, R. Gunawidjaja, R. R. Naik, and V. V. Tsukruk, "pH-Triggered SERS via Modulated Plasmonic Coupling in Individual Bimetallic Nanocobs," Small 7, 1192-1198 (2011).

47. Y. Roiter, I. Minko, D. Nykypanchuk, I. Tokarev, and S. Minko, "Mechanism of nanoparticle actuation by responsive polymer brushes: from reconfigurable composite surfaces to plasmonic effects," Nanoscale 4, 284-292 (2012).

48. A. Abbas, M. Fei, L. M. Tian, and S. Singamaneni, "Trapping Proteins within Gold Nanoparticle Assemblies: Dynamically Tunable Hot-spots for Nanobiosensing," Plasmonics 8, 537-544 (2013).

49. X. Liu, X. Wang, L. Zha, D. Lin, J. Yang, J. Zhou, and L. Zhang, "Temperature- and $\mathrm{pH}$-tunable plasmonic properties and SERS efficiency of the silver nanoparticles within the dual stimuli-responsive microgels," Journal of Materials Chemistry C 2, 7326-7335 (2014).

50. W. Lewandowski, M. Fruhnert, J. Mieczkowski, C. Rockstuhl, and E. Gorecka, "Dynamically self-assembled silver nanoparticles as a thermally tunable metamaterial," Nat Commun 6 (2015).

51. J. Lee, S. Yoo, M. Shin, A. Choe, S. Park, and H. Ko, "pHtunable plasmonic properties of Ag nanoparticle cores in block copolymer micelle arrays on Ag films," Journal of Materials Chemistry A 3, 11730-11735 (2015).

52. I. Tokarev, and S. Minko, "Tunable plasmonic nanostructures from noble metal nanoparticles and stimuli-responsive polymers, " Soft Matter 8, 5980-5987 (2012).

53. R. W. Carpick, and M. Salmeron, "Scratching the Surface: Fundamental Investigations of Tribology with Atomic Force Microscopy," Chem. Rev. 97, 1163-1194 (1997).

54. P. A. Bobbert, and J. Vlieger, "Light scattering by a sphere on a substrate," Physica A 137, 209-242 (1986).

55. B. R. Johnson, "Calculation of light scattering from a spherical particle on a surface by the multipole expansion method," J. Opt. Soc. Am. A 13, 326-337 (1996).

56. E. Fucile, P. Denti, F. Borghese, R. Saija, and O. I. Sindoni, "Optical properties of a sphere in the vicinity of a plane surface," J. Opt. Soc. Am. A 14, 1505-1514 (1997).

57. E. Fucile, F. Borghese, P. Denti, R. Saija, and O. I. Sindoni, "General reflection rule for electromagnetic multipole fields on a plane interface," Antennas and Propagation, IEEE Transactions on 45, 868-875 (1997).

58. M. I. Mishchenko, G. Videen, V. A. Babenko, N. G. Khlebtsov, and T. Wriedt, "T-matrix theory of electromagnetic scattering by particles and its applications: a comprehensive reference database," J. Quant. Spectros. Radia. Transfer 88, 357-406 (2004).

59. M. I. Mishchenko, Electromagnetic Scattering in Discrete Random Media:Fundamental Theory and Applications (Cambridge University Press, 2002).

60. W. Y. Chien, and T. Szkopek, "Multiple-multipole simulation of optical nearfields in discrete metal nanosphere assemblies," Opt. Express 16, 1820-1835 (2008).

61. J. Parsons, C. P. Burrows, J. R. Sambles, and W. L. Barnes, "A comparison of techniques used to simulate the scattering of electromagnetic radiation by metallic nanostructures," Journal of Modern Optics 57, 356-365 (2010).

62. G. Mie, "Beiträge zur Optik trüber Medien, speziell kolloidaler Metallösungen," Annalen der Physik 330, 377445 (1908).

63. P. B. Johnson, and R. W. Christy, "Optical Constants of the Noble Metals," Phys. Rev. B 6, 4370-4379 (1972).

64. S. Raza, S. I. Bozhevolnyi, M. Wubs, and N. A. Mortensen, "Nonlocal optical response in metallic nanostructures," J. Phys. Condens. Matter 27, 183204 (2015).

65. I. Ashry, B. Zhang, S. V. Stoianov, C. Daengngam, J. R. Heflin, H. D. Robinson, and Y. Xu, "Probing the photonic density of states using layer-by-layer self-assembly," Opt. Lett. 37, 1835-1837 (2012).

66. C. D. Bain, E. B. Troughton, Y. T. Tao, J. Evall, G. M. Whitesides, and R. G. Nuzzo, "Formation of Monolayer Films by the Spontaneous Assembly of Organic Thiols from Solution onto Gold," J. Am. Chem. Soc. 111, 321-335 (1989).

67. Z. Feldötö, M. Lundin, S. Braesch-Andersen, and E. Blomberg, "Adsorption of IgG on/in a PAH/PSS multilayer film: Layer structure and cell response," J. Colloid Interface Sci. 354, 31-37 (2011).

68. J. E. Wong, F. Rehfeldt, P. Hänni, M. Tanaka, and R. v. Klitzing, "Swelling Behavior of Polyelectrolyte Multilayers in Saturated Water Vapor," Macromol. 37, 7285-7289 (2004).

69. H. Shibru, Y. Zhang, K. L. Cooper, G. R. Pickrell, and A. Wang, "Optimization of layer-by-layer electrostatic selfassembly processing parameters for optical biosensing," Optical Engineering 45, 024401 (2006).

70. N. Granqvist, H. Liang, T. Laurila, J. Sadowski, M. Yliperttula, and T. Viitala, "Characterizing Ultrathin and Thick Organic Layers by Surface Plasmon Resonance Three-Wavelength and Waveguide Mode Analysis," Langmuir 29, 8561-8571 (2013). 
71. H. Hövel, S. Fritz, A. Hilger, U. Kreibig, and M. Vollmer, "Width of cluster plasmon resonances: Bulk dielectric functions and chemical interface damping," Phys. Rev. B 48, 18178-18188 (1993).

72. B. Foerster, A. Joplin, K. Kaefer, S. Celiksoy, S. Link, and C. Sönnichsen, "Chemical Interface Damping Depends on Electrons Reaching the Surface," ACS Nano 11, 2886-2893 (2017).

73. S. V. Stoianov, C. Daengngam, M. Borhani, Y. Zhang, J. R. Morris, and H. D. Robinson, "Amine-Rich Polyelectrolyte Multilayers for Patterned Surface Fixation of Nanostructures," ACS Appl. Mat. Interfaces 4, 2348-2357 (2012).

74. F. Caruso, K. Niikura, D. N. Furlong, and Y. Okahata, "1. Ultrathin Multilayer Polyelectrolyte Films on Gold: Construction and Thickness Determination," Langmuir 13, 3422-3426 (1997).

75. B.-S. Kim, and O. I. Vinogradova, "pH-Controlled Swelling of Polyelectrolyte Multilayer Microcapsules," J. Phys. Chem. B 108, 8161-8165 (2004).

76. G.-Y. Liu, S. Xu, and Y. Qian, "Nanofabrication of SelfAssembled Monolayers Using Scanning Probe Lithography," Acc. Chem. Res. 33, 457-466 (2000).

77. H. Gong, J. Garcia-Turiel, K. Vasilev, and O. I. Vinogradova, "Interaction and Adhesion Properties of Polyelectrolyte Multilayers," Langmuir 21, 7545-7550 (2005).
78. T. Pettersson, S. A. Pendergraph, S. Utsel, A. Marais, E. Gustafsson, and L. Wågberg, "Robust and Tailored Wet Adhesion in Biopolymer Thin Films," Biomacromolecules 15, 4420-4428 (2014).

79. R. Lingström, S. M. Notley, and L. Wågberg, "Wettability changes in the formation of polymeric multilayers on cellulose fibres and their influence on wet adhesion," J. Colloid Interface Sci. 314, 1-9 (2007).

80. A. J. Nolte, J. Y. Chung, M. L. Walker, and C. M. Stafford, "In situ Adhesion Measurements Utilizing Layer-by-Layer Functionalized Surfaces," ACS Appl. Mat. Interfaces 1, 373 380 (2009).

81. J. J. Mock, M. Barbic, D. R. Smith, D. A. Schultz, and S. Schultz, "Shape effects in plasmon resonance of individual colloidal silver nanoparticles," J. Chem. Phys. 116, 67556759 (2002).

82. A. Csaki, T. Schneider, J. Wirth, N. Jahr, A. Steinbrück, O. Stranik, F. Garwe, R. Müller, and W. Fritzsche, Molecular plasmonics: light meets molecules at the nanoscale 369, 3483-3496 (2011).

83. N. Meinzer, W. L. Barnes, and I. R. Hooper, "Plasmonic meta-atoms and metasurfaces," Nat. Photonics 8, 889-898 (2014).

84. N. I. Zheludev, and Y. S. Kivshar, "From metamaterials to metadevices," Nat. Mater. 11, 917-924 (2012). 